\title{
Mitigating the effect of errors in source parameters on seismic (waveform) inversion
}

\author{
Blom, Nienke (nienke.blom@esc.cam.ac.uk) \\ Hardalupas, Phil-Simon (psh \\ Rawlinson, Nicholas (nr411@cam.ac.uk)
}

This paper is a non-peer reviewed preprint submitted to EarthArXiv, and has been submitted for peer review at Geophysical Journal International, where it is now under revision. 
submitted to Geophys. J. Int.

\title{
Mitigating the effect of errors in source parameters on seismic (waveform) tomography
}

\author{
Nienke Blom ${ }^{1}$ and Nicholas Rawlinson ${ }^{1}$ \\ ${ }^{1}$ Bullard Laboratories, Department of Earth Sciences, University of Cambridge, Madingley Rise, Cambridge CB3 OEZ,
}

2 September 2021

\section{SUMMARY}

We investigate the effect of errors in earthquake source parameters on the tomographic inverse problem and propose mitigation strategies for avoiding artefacts caused by such errors. Source errors can be quite significant: mislocation errors can amount to tens of kilometres both horizontally and vertically, while fault plane uncertainties can be of the order of tens of degrees. To analyse how these errors affect seismograms and hence seismic tomography, we conduct a perturbation study involving a number of synthetic inversions that feature several types of source errors. It is well known that source location errors can detrimentally influence the recovery of seismic structure, but we find that also errors in the moment tensor can give rise to significant errors: even those moment tensor components to which computed waveforms are least sensitive can result in significant wavefield perturbations and corresponding timeshifts. The effect of such errors on tomographic images is consequently pronounced. All source error types that we investigate result in considerable artefacts in recovered Earth models, which for realistic errors are similar in amplitude to what is typically imaged using observational data. In some cases these artefacts extend over thousands of kilometres.

We present a number of mitigation strategies to minimise the formation of source error artefacts in tomographic images. By removing a region around the source and discarding measurements on or near nodal planes (where amplitudes are small), the adverse effects of many types of source error can be greatly reduced. The most problematic type of error is a horizontal mis- 


\section{N.A.Blom, N. Rawlinson}

location, because its imprint remains visible even when taking such mitigation measures into account. Moreover, its effect cannot be easily distinguished from the effect of several other types of source errors or in fact from any Earth structure that has a degree-2 pattern with respect to the source. Horizontal event location uncertainty must therefore be carefully evaluated, and preferably compared to 'ground truth' data where this is available. Failing that, suspicious events may need to be removed altogether from the inverse problem in order to avoid their imprint contributing significantly to the imaged structure.

Although this study focuses on (adjoint) waveform tomography, a large part of the results are equally valid for any other type of imaging method that is based on time- and/or phaseshift measurements. We therefore expect that the concerns and mitigation strategies presented here have broader applicability.

Key words: Seismology, seismic tomography, earthquake parameters, inverse theory.

\section{INTRODUCTION}

Earthquakes are a primary source of information that can be exploited to gain knowledge about the Earth's interior. The elastic waves they emit are used in seismic tomography to image the 3-D structure of the Earth. At a local scale, they are complex ruptures that may develop along different branches of faults over an extended period of time. As a result of this, energy radiates outwards from the source in complex patterns that vary as a function of space and time. Describing or modelling these processes in detail requires significant effort and resources, which need to be applied to every earthquake individually (e.g. Simons et al. 2011; Ulrich et al. 2019; Wollherr et al. 2019). It is therefore not surprising that the determination of earthquake rupture parameters from seismic records is a very non-unique inverse problem.

Seismic tomography, in contrast, works at much larger scales. The area to be imaged is typically orders of magnitude larger than the source region and receivers are generally located many wavelengths from the source (Nolet 2008). Because of this, earthquakes can usually be considered point sources that are described sufficiently well with a single spatial position, an origin time and possibly a description of the spatial radiation of energy. In most tomographic contexts, at most ten 
parameters are thus necessary to describe an earthquake (Dziewoński et al. 1981): three spatial coordinates, an origin time and the symmetric moment tensor which describes the energy radiation. Many different catalogues exist which routinely supply such earthquake parameters (e.g. Ekström et al. 2012; Masse \& Needham 1989), which are commonly used as prior information in the imaging procedure.

Strictly speaking, a source description is only valid within the exact framework in which it was derived, i.e. the same Earth model, modelling method, dataset and processing procedure (see Valentine \& Trampert 2012). The Earth model in particular is likely to differ substantially between a catalogue source inversion and a tomographic application. Moreover, the model changes as the tomographic inversion progresses. Ideally, source and structure are therefore inverted for together in order to ensure consistency (Pavlis \& Booker 1980; Spencer \& Gubbins 1980; Thurber 1992; Valentine \& Woodhouse 2010; Lu \& Grand 2016).

If the sources are located within the domain of interest, traveltime tomography studies typically incorporate joint or iterative source-structure inversion procedures (e.g. Aki \& Lee 1976; Pavlis \& Booker 1980; Thurber 1983; Dziewoński 1984; Engdahl \& Gubbins 1987; Inoue et al. 1990; Bijwaard et al. 1998; Moulik \& Ekström 2016). For teleseismic tomography, the effect of errors in the source is largely removed by subtracting the mean of arrival time differentials for each sourcereceiver set, although this also makes the method largely insensitive to radial velocity structure. In some waveform tomography studies, source parameters are also inverted for at various stages of the inversion (e.g. Tape et al. 2009; Fichtner et al. 2009, 2013).

Often, however, source parameters are omitted entirely from the inverse problem. This is done for a variety of reasons: it can be technically challenging or computationally unfeasible to implement a joint inversion strategy, there may be issues with (azimuthal) data coverage, or it increases trade-offs/non-uniqueness to an unacceptable level. This is further exacerbated by the fact that the moment tensor components are linearly related to seismograms (see Section 2.1), whereas the spatial parameters have complicated non-linear relationships. In traveltime tomography, the velocity inversion is also much less non-linear than the source location problem. Such imbalances can make trade-offs difficult to deal with. As a result of such complications, source informa- 


\section{4}

N.A.Blom, N. Rawlinson

tion is often taken directly from catalogues (e.g. Dziewoński et al. 1977; Lekić \& Romanowicz 2011; Ma et al. 2014; Simute et al. 2016; Krischer et al. 2018; van Herwaarden et al. 2020), corrected/relocated/reinverted once using the starting model (e.g. Grand 2002; Zhu et al. 2012; Bozdağ et al. 2016), or manually adjusted on an ad hoc basis (e.g. Blom et al. 2020, - see their Supplementary Material for details).

Indeed, we have found in previous work that adjustments to the source parameters can have a significant effect on data fit in the context of waveform tomography, as demonstrated in Figure 1. In an example taken from Blom et al. (2020), an event located at the original catalogue position results in a clear azimuthal pattern of phase shifts (Figure 1a). This pattern can be removed by shifting the event to the north-east by $10 \mathrm{~km}$ (Fig. 1b). It is clear that, if indeed the pattern is due entirely to an erroneous source location, this corrupts the phase shifts generated by 3-D structure. Adjustments like the one demonstrated in Figure 1 are possible in cases where one has some degree of confidence that the pattern is indeed the result of a specific error in the source - but based only on the measurements, it is impossible to distinguish between a horizontal shift and any structural heterogeneity that has a degree-2 pattern. It should also be noted that such measures obviously introduce a certain level of bias into the inverse problem, and it cannot be expected that all source errors can be detected using such ad-hoc methods. As long as source errors are randomly distributed, they are likely to balance out in areas where many sources are present. However, the effects of systematic errors in catalogue parameters accumulate in the tomographic modelling, thereby compromising the robustness of the imaging results. This is likely to be an issue in many tomographic settings.

A few studies have investigated the effect of erroneous source parameters on seismic tomography. Valentine \& Woodhouse (2010) find that erroneous source locations have a significant effect on recovered Earth structure, with artefacts often similar in amplitude to actual model anomalies. Importantly, tomography models recovered using fixed sources contain an imprint of the Earth model used in the source inversion. Lu \& Grand (2016) focus on small-scale subduction zone structure, finding that source mislocations typical for subduction zones have the effect of largely removing the slab signal itself, while also resulting in artefacts far away from the subduction zone. 
a.

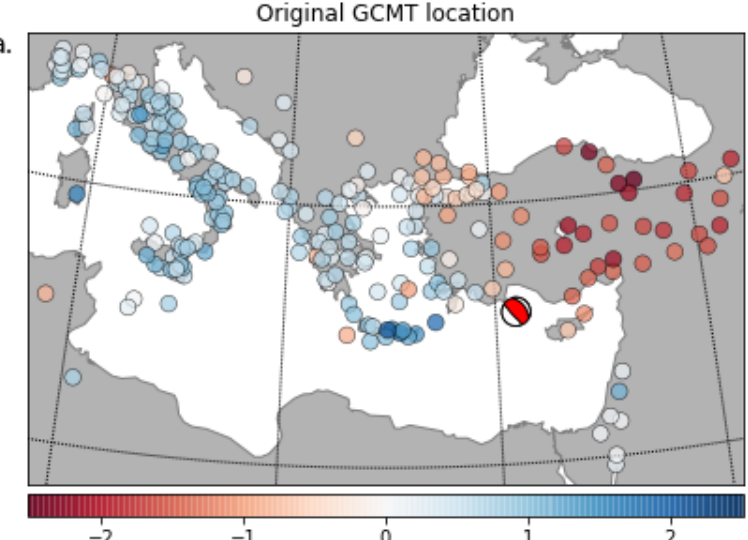

$-2$

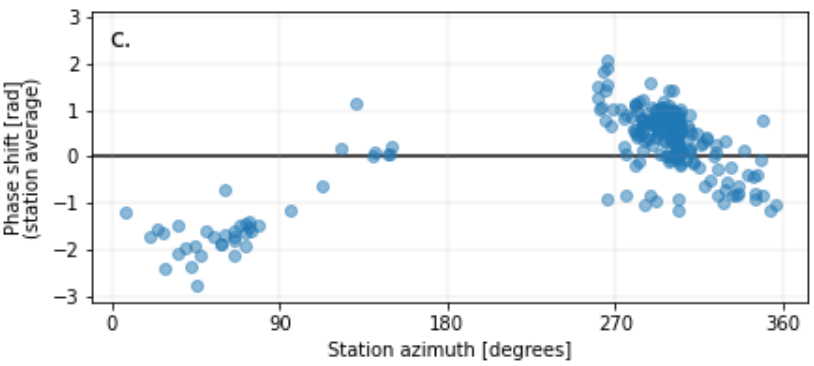

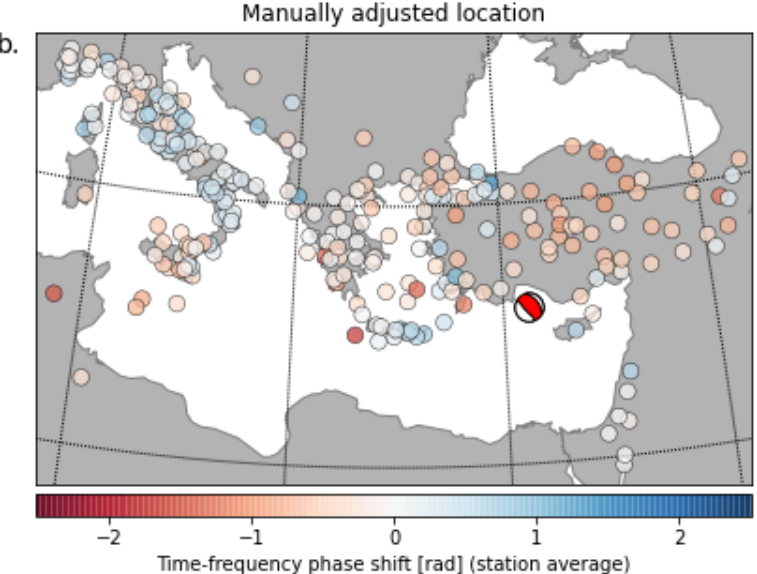

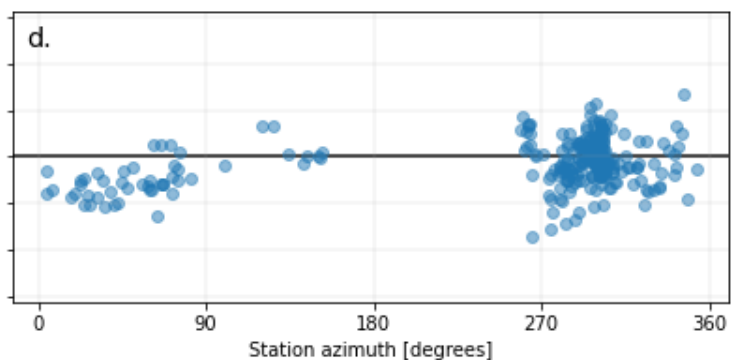

Figure 1. A demonstration of the effect of an event relocation using real-world data. Data taken from Blom et al. (2020). Shown is the time-frequency phase shift (Fichtner et al. 2008, see Equation 7) between observed and predicted seismograms. (a) The initial event parameters (taken from the GCMT catalogue) result in a clear azimuthal pattern of phase shifts. (b) A manual shift towards the north-east mostly removes this azimuthal pattern, and the remaining phase shifts are much smaller in amplitude. (c) Phase shifts as a function of azimuth for the original GCMT event location, which reveal a sinusoidal pattern. (d) Phase shifts as a function of azimuth for the manually adjusted location. All phase shifts are now closer to zero, and the sinusoidal pattern is no longer evident.

Yamaya et al. (2018) investigated the effect of redetermining the source-time-functions of deep and intermediate earthquakes in the recovery of 3-D structure in the D" region using a variant of waveform inversion, and found that the effect was mostly limited to the amplitude of the recovered structure. In contrast, Ma \& Masters (2015) argue that realistic source location errors have a limited effect on inverted isotropic velocity structure, although they need to be taken into account when inverting for azimuthal anisotropy. However, these results are based on very smooth models.

In this paper, we methodically investigate the effect of errors in source parameters on seismic measurements and consequently in tomographic inverse problems, with a special focus on waveform tomography. So far, the influence of source errors on tomographic results has received limited 


\section{6}

N.A.Blom, N. Rawlinson

attention (Valentine \& Woodhouse 2010; Ma \& Masters 2015; Lu \& Grand 2016; Yamaya et al. 2018), and there are no studies that systematically evaluate the effect of such errors on waveform tomography in particular.

After a review of the relevant theory, we first present a literature overview of errors likely to be found in source parameters and the extent to which these may be problematic in a tomography context (Section 3). Building on this context, we systematically analyse how perturbations in the source change the resulting seismograms, and as a consequence, the measurements made on them that are typically used for tomography (Section 5). We further assess the extent to which this affects the recovered model in seismic tomography (Section 6).

\section{THEORY}

Because the scale at which seismic tomography is conducted is typically much larger than the scale of source processes, a number of approximations and assumptions can be made that greatly reduce the number of parameters needed to describe an earthquake with sufficient accuracy. The most important assumption is the point source approximation, which is valid as long as the source rupture is small in size compared to the wavelengths used in the analysis and the propagation distance between source and receiver (Udías et al. 2014).

Depending on the type of information used in the tomography, additional simplifications to the source description can be made. In travel-time tomography, for instance, information on the spatial radiation pattern of energy is not needed, as the only information that is recorded from seismograms is the arrival time of certain phases. The radiation pattern only influences their polarity or visibility. Waveform tomography, in contrast, relies on accurately matching observed and synthetic seismograms, and thus needs an accurate description of the spatial radiation of energy.

While the source-time function can play a large role in higher-frequency applications (Yamaya et al. 2018), most waveform tomography studies operate at periods that are much longer than the source duration of the events that are used. For suitably chosen events, earthquakes can thus be considered point sources in time as well as space.

It should be noted that this remains an assumption: while rupture area and length broadly scale 
with earthquake magnitude, they can easily vary by an order of magnitude for similar-sized events (see Wells \& Coppersmith 1994; Somerville et al. 1999; Strasser et al. 2010). The same holds for source duration: Vallée et al. (2011) cite the example of two M7.7 earthquakes where one lasts 15 s and the other 150 s (see also Stein \& Pelayo 1991; Tanioka \& Ruff 1997).

\subsection{The moment tensor}

The spatial radiation of energy emitted from an earthquake can be described using the concept of moment tensors (Gilbert 1971). Based on the notion of generalised force couples, it can be used to describe shear motion on a fault surface (a double couple), but also sources involving volume change or tensile cracks (see e.g. Knopoff \& Randall 1970; Jost \& Herrmann 1989). A moment tensor is a symmetric second-order tensor $\boldsymbol{M}$ where each component $M_{i j}$ describes a pair of forces pointing in the direction $i$ and separated by an infinitesimal distance in the direction $j$. Several different coordinate systems are in use for the description of the moment tensor. Here we will use the common Up-South-East description:

$\boldsymbol{M}=\left(\begin{array}{ccc}M_{r r} & M_{r \theta} & M_{r \phi} \\ M_{\theta r} & M_{\theta \theta} & M_{\theta \phi} \\ M_{\phi r} & M_{\phi \theta} & M_{\phi \phi}\end{array}\right)$,

where $r$ is the radial direction (Up), $\theta$ corresponds to South and $\phi$ corresponds to East.

The moment tensor is symmetric and its components are linearly independent, each having characteristic radiation patterns for P-, S- and surface waves. Several examples of moment tensors and the resulting radiation of energy are included in Supplementary Figure S2. The seismic moment $M_{0}$ can be described by

$M_{0}=\frac{1}{\sqrt{2}}\left(M_{i j}\right)^{\frac{1}{2}}$,

and relates to the moment magnitude $M_{W}$ through

$M_{W}=\frac{2}{3} \log _{10}\left(M_{0} \cdot 10^{7}\right)-10.7$,

where the seismic moment is given in Nm (Hanks \& Kanamori 1979). 
The displacement at a location $x_{r}$ due to an impulse force $f$ at location $x_{s}$ can be described as

$u_{i}\left(x_{r}\right)=G_{i j}\left(x_{r} ; x_{s}\right) * f_{j}$,

where $G$ is a Green's function or impulse response (Shearer 2019). Because each component $M_{i j}$ represents a force couple, it follows that the moment tensor components and the displacement of the Earth at $x_{r}$ are linearly related.

The classical description of Knopoff \& Randall (1970) is often used to decompose a source into physically meaningful parts: a component relating to explosive (or implosive) behaviour (the isotropic part), one that describes slip along a plane (the double couple), and the 'remainder' CLVD, which may indicate the presence of tensile cracks but is often interpreted as an artefact resulting from the absorption of unmodelled structure (e.g. ignoring lateral heterogeneity), or the superposition of several sub-events. This decomposition is not unique, and many others exist (see e.g. Kanamori \& Given 1981; Jost \& Herrmann 1989; Krieger \& Heimann 2012; Vavryčuk 2015).

A pure DC source can be described uniquely by only three parameters. One parametrisation describes the strike, dip and rake for one of the planes (the other plane follows from that, and so does the moment tensor). In contrast to the moment tensor components, this parametrisation is related non-linearly to the wavefield, but its direct correspondence to fault planes is an advantage. A description of the relationship between such fault plane solutions and the moment tensor components is given in e.g. Henry et al. (2002) and numerous algorithms exist that compute the conversion in both directions (e.g. the MoPaD toolbox of Krieger \& Heimann 2012, which is included in the ObsPy package).

In the spatio-temporal point source approximation, an earthquake can generally be described by ten parameters: a 3-D location, an origin time, and six moment tensor components. While the relationship between ground displacement and moment tensor components is linear, the relationship with 3-D location is not. This means that inverting for source parameters normally requires an iterative approach. 


\subsection{Determining earthquake parameters: GCMT}

The determination of earthquake parameters has received a lot of attention due to its importance in not only tomography, but a wide range of other applications such as seismic hazard assessment. As a consequence, a large number of methods and catalogues exist, which are often tailored to a specific context and/or purpose. One of the most widely-used catalogues comes from the Global Centroid Moment Tensor (GCMT) project (Ekström et al. 2012; Dziewoński et al. 1981, , http://www.globalcmt.org), because it supplies both moment tensors and locations. It is based on long-period body and surface wave data $(\mathrm{T}>=40 \mathrm{~s})$ and makes use of the waveform matching of global seismic records. It is therefore the catalogue of choice for most waveformbased applications, which tend to use a similar period band. We briefly summarise the algorithm below, but refer to Ekström et al. (2012) for the full details.

The GCMT algorithm works semi-automatically, using three different datasets that are weighted according to the event's magnitude: long-period body waves (40-150 s), intermediate period surface waves (50-150 s) and long-period surface waves referred to as 'mantle waves' (125-450 s). The choice of band-pass filter applied, and whether displacement or velocity data is used, depends on the event magnitude. Inversion for the source parameters takes place using a nested iterative approach, whereby data selection and inversion are carried out sequentially until the source parameters stabilise. This yields a centroid, i.e. the average location in space and time of the energy release and a corresponding moment tensor. This is different from the hypocentre, the point where rupture started, but in the following we will use these terms somewhat interchangeably.

Some additional constraints are included in the inversion. Because of limited sensitivity of the data to the full moment tensor, the isotropic component of the source is assumed to be zero, that is, the inversion assumes that $M_{r r}+M_{\phi \phi}+M_{\theta \theta}=0$ (Dufumier \& Rivera 1997). Because of the relatively long periods used, depth is also poorly resolved, especially in the case of shallow events. As a result, GCMT depths are kept at a minimum of $12 \mathrm{~km}$, and in some cases the depths of deeper events are also fixed, either at the originally reported value (usually the NEIC catalogue - see Masse \& Needham 1989) or at a depth ‘deemed appropriate for the source region' (Ekström et al. 2012). The source half-duration is derived from the earthquake's scalar moment using an 
empirical relationship and the source-time function is modelled as an isoscles triangle (as a boxcar before 2004).

Synthetic data for the three data types used in the inversion are calculated in different ways. The body waves and mantle waves are computed through normal mode summation, as are the overtones of the intermediate period surface waves. These synthetics are corrected for the effects of threedimensional structure (Dziewoński et al. 1984). The fundamental mode intermediate surface wave synthetics are computed using a surface-wave ray-based method which incorporates phase delays predicted by global dispersion maps (Ekström et al. 1998).

The procedure is carried out in a least squares formalism which also yields the associated leastsquares uncertainties. These uncertainties are based on the assumption that the only source of error is in the form of uncorrelated data noise with a Gaussian distribution, which is strictly only valid within the particular framework used in the GCMT source inversion procedure. Thus potential modelling errors/biases are not taken into account (Smith \& Ekström 1997; Ekström 2011; Valentine \& Trampert 2012), whether from an erroneous Earth model, from the waveform modelling method, or from other assumptions made within the design of the source inverse problem.

\subsection{Other methods for determining source parameters}

In addition to GCMT, there are other regional and global moment tensor catalogues that use different approaches to constraining the source mechanism. For example, the National Earthquake Information Centre (NEIC), which is part of the United States Geological Survey (USGS) has routinely been computing moment tensor solutions of moderate to large-sized earthquakes since 1981 (Sipkin 2002). The inversion method is based on multichannel signal enhancement theory and exploits P-waveform data, which distinguishes it from the GCMT approach. Only data from an epicentral distance range of $30-95^{\circ}$ are used, and the solutions are constrained to be purely deviatoric, rather than purely double couple (Sipkin 2002). Due to the incorporation of depth-sensitive phases such as pP and sP the method has the potential to accurately constrain depth, though it is limited by the use of a 1-D global reference model (ak135, Kennett et al. 1995), which has limited applicability in complex tectonic settings. 
Other moment tensor catalogues also exist, but these tend to be computed by national agencies, and are therefore regional in nature, and use a range of methods. For example, the ANSS Comprehensive Earthquake Catalogue (ComCat) of the USGS contains earthquake source parameters from a range of contributing seismic networks. More boutique catalogues also exist, such as gWFM (the Global Waveform-Modelled Earthquake catalogue), which only contains high-fidelity mechanisms and centroid depths from moderate-magnitude earthquakes between 1915-2019 (Wimpenny \& Watson 2021). This is achieved through body waveform modelling of broadband data, which can offer good depth constraints for moderate-size events. However, this catalogue, too, relies on the quality of the supplied 1-D model and takes the locations from the (ISC-)EHB catalogue, which means that the combined parameters are not necessarily self-consistent.

There are of course many national and global catalogues that only constrain source location, origin time and magnitude. Although of limited value in waveform tomography, these can be very useful in ray-based studies such as traveltime tomography. The authorative global earthquake catalogue is provided by the International Seismology Centre (ISC), and is known as the ISC Bulletin, which contains data from 1900 onwards. It relies on data, including phase picks, from seismological agencies from around the world. Due to the inconsistencies in data that comes from a diverse range of sources, the ISC-EHB catalogue (Storchak et al. 2015) offers a much more groomed set of earthquakes, that have been located using strict criteria, as described in Engdahl et al. (1998) and Engdahl (2006); this includes a specific algorithm for treating depth-sensitive phases, resulting in improved depth estimates compared to the basic ISC bulletin. The ISC-EHB and its predecessor the EHB bulletin have been successfully used in regional and global seismic traveltime tomography (e.g. Amaru 2007; Zenonos et al. 2019). Other global earthquake catalogues such as NEIC PDE are also available, but since they are ultimately incorporated in the ISC Bulletin, we do not discuss them further here.

\section{ERRORS IN SOURCE PARAMETERS: AN OVERVIEW}

Errors in earthquake parameters can have a variety of causes. Data noise is an important factor and can be caused by oceanic microseisms, wind and local sources. Human error in the form 
of mis-picking and phase misidentification can also corrupt the data, as well as station issues to do with timing, location, orientation, response function, or instrument problems, etc. These issues may or may not be systematic, depending on the root cause. The way that the inverse problem is formulated and solved generally contains a variety of assumptions that also feed into the uncertainty associated with the parameters that are constrained. Such assumptions include the source parametrisation itself (such as the point source approximation), but also a fixed depth or magnitude-derived source duration. Finally, errors can be caused by the way in which the synthetic data are computed, regardless of whether these are travel times or waveforms. This may be a result of the modelling method itself, but can also derive from the Earth model used.

We note here the distinction between errors which are geologically relevant (say, the exact dip of a fault plane or the depth of an earthquake) and those that affect tomography. For example, it is well-known that the precise depth of an earthquake has little effect on long-period seismograms in particular; as long as both the source inversion and the tomographic application use such long-period seismograms, the precise depth does not the results. Some source errors are thus less relevant for tomography than others, and the same holds for trade-offs between parameters. Uncertainties for moment tensor components derive from the magnitude of their Green's function. If this is small, the effect of the component on a seismogram is correspondingly small. If this holds for many or all seismograms used in an inversion for source parameters, the uncertainty associated with this component is correspondingly large, and small errors or noise in the data can produce spuriously large values.

Source parameters can differ significantly depending on the Earth model used in their determination. Several authors have investigated the 'likely' errors that can be expected from catalogue source parameters, finding even for smooth models hypocentral uncertainties of the order of tens of kilometres, as well as significant changes to moment tensors that correspond to fault plane uncertainties of tens of degrees (Smith \& Ekström 1996; Ferreira \& Woodhouse 2006; Valentine \& Woodhouse 2010; Hjörleifsdóttir \& Ekström 2010; Valentine \& Trampert 2012). These errors become more pronounced when models are used that are more strongly heterogeneous (Hejrani et al. 2017). Below, we discuss different types of errors that may be present in the source parameters. 
We will focus on the 10-parameter spatio-temporal point source description, as used in the GCMT catalogue. As well as errors in single parameters, trade-offs can exist between source parameters, i.e. correlated errors.

\subsection{Horizontal location}

The determination of the horizontal location, or epicentre, has long been known to be affected by the Earth model used for the source determination - regardless of the modelling approach. Smith \& Ekström (1996) derived hypocentre locations in different Earth models for a set of 'known' explosions and earthquakes, based on teleseismic travel-time arrival times. They find that 3-D Earth structure has significant influence on the derived source location (typically 10-20 km), an effect that is likely to be more pronounced in regions such as subduction zones where significant small-scale lateral heterogeneity exists, which is not incorporated in smooth global models. Other studies report similar values (e.g. Shearer 2001; Craig 2019), with significant regional variability depending on Earth structure and station coverage.

This is also true for the GCMT algorithm: Dziewoński \& Woodhouse (1983) already pointed out that lateral heterogeneities can introduce a substantial bias $\left(1^{\circ}\right.$ longitude or $\sim 85 \mathrm{~km}$ for their Northern California example). Although the GCMT algorithm includes corrections for 3-D structure, these are based on smooth models, and the GCMT team themselves stress that the epicentre in particular may not reflect the 'true' position of the source, and is necessarily a function of the Earth model used (Dziewoński et al. 1987). Studies using smooth, global models find epicentral errors for GCMT source mechanisms of the order of $10 \mathrm{~km}$ (Ferreira \& Woodhouse 2006; Hjörleifsdóttir \& Ekström 2010; Valentine \& Trampert 2012), while the more strongly heterogeneous waveform tomography model used by Hejrani et al. (2017) results in significantly larger shifts (up to $72 \mathrm{~km}$ ). It is thus likely that for the real Earth, a reasonable estimate of epicentral location errors would be $20-40 \mathrm{~km}$.

Systematic shifts, in this context, are of particular concern, especially in areas displaying strong lateral heterogeneity such as subduction zones (Shearer 2001; Hjörleifsdóttir \& Ekström 2010; Lu \& Grand 2016). Figure S1 illustrates this for the North Chilean subduction zone, using a dataset 
collected by Craig (2019). Comparing horizontal locations from the GCMT catalogue with those from ISC-EHB, EHB and NEIC catalogues, a systematic trench-perpendicular shift is visible beween the two, typically of the order of $20-40 \mathrm{~km}$ (Figure S1b). Given the magnitudes of the events (typically between $\mathrm{M}_{W} 5$ and 6), this is unlikely to be a result of the rupture size, and is more likely caused by the different Earth models used. The effect of such systematic errors on tomography can be considerable (Lu \& Grand 2016).

\subsection{Depth}

The most reliable way of determining the depth of an earthquake is through the detection of depth phases (Engdahl et al. 1998; Craig 2019), which works best for deeper events where these phases are properly separated from the initial arrival. Another method, more suitable for shallow events, is based on waveform modelling of body waves (see Wimpenny \& Watson 2021, for a discussion). Even in those cases, the derived source depth is dependent on near-source Earth structure and depth thus remains a poorly determined parameter. Compared to other methods, GCMT depths are relatively poorly constrained because of the comparatively long-period data used - in particular at shallow depths where they tend to be fixed at $12 \mathrm{~km}$. A well-known trade-off exists between depth and origin time (Nolet 2008), and inferred depths may also be influenced by trade-offs with source duration, where depth phases can be mistaken for a long source time function, or vice versa (Dufumier \& Rivera 1997; Vallée et al. 2011).

For the relatively smooth models used in their analysis, Hjörleifsdóttir \& Ekström (2010) find GCMT depth errors to be on the order of $10 \mathrm{~km}$, similar to the horizontal errors, while Hejrani et al. (2017) obtain differences of the order of 10-20 km. Depth is most strongly influenced by near-source and near-receiver structure - if this is accounted for in the inversion, this error can be reduced significantly (Hjörleifsdóttir \& Ekström 2010). A systematic shift in depths may also be introduced in regions with anomalous crustal structure.

Supplementary Figure S1c and d compare depths from the GCMT catalogue with those from the local catalogue derived by Craig (2019), who uses a semi-automated stacking procedure to determine accurate earthquake depths based on multiple depth phases and an accurate local veloc- 
ity model. The systematic depth shift is typically 5-15 km, with GCMT generally giving deeper values. The refined catalogue of Craig (2019) shows significantly less scatter, indicating that the results are more self-consistent.

\subsection{Origin time}

Origin time errors are likely to be of the order of at least a few seconds for moderate-magnitude events (e.g. Ferreira \& Woodhouse 2006; Hejrani et al. 2017). If the shallow structure in an area differs substantially from the model used in source determination, systematic shifts in origin time can occur. This is visible in the results of Hjörleifsdóttir \& Ekström (2010), who report a systematic error in their inverted origin times, which are on average 2 seconds later than the true values - a bias which is strongest for the surface wave data. For body waves, origin time and event depth display a trade-off that depends on the radial velocity model (Pavlis \& Booker 1980; Spencer \& Gubbins 1980), but trade-offs with Earth structure exist in any direction and are a function of heterogeneity strength (Hejrani et al. 2017).

\subsection{Moment tensor errors}

The GCMT reported errors in the moment tensor components are based on the assumption that only data noise contributes to them. Valentine \& Trampert (2012) determine realistic error ranges for each of the moment tensor components and find these are of the order of $15-50 \%$ of the event's seismic moment. This can translate into uncertainties in fault plane orientations of tens of degrees.

In particular, the $M_{r \theta}$ and $M_{r \phi}$ components are poorly constrained, which together represent pure dip-slip on an arbitrary vertical plane or shear motion on an arbitrary horizontal plane. These components have Green's functions that vanish at the Earth's surface, and thus become very unstable if the earthquake depth is only a small fraction of the wavelengths used in the source inversion. For shallow dip-slip earthquakes, this results in a trade-off between the seismic moment $M_{0}$ and the $\operatorname{dip} \delta$, as the excitation of surface waves for a dip-slip source is proportional to $M_{0} \sin (2 \delta)$ (Kanamori \& Given 1981). Dziewoński \& Woodhouse (1983) point out that the effect of lateral heterogeneity can also be easily absorbed by an artificial contribution to these components - re- 
sults confirmed by Henry et al. (2002) and Ferreira \& Woodhouse (2006), the latter finding errors of up to $80 \%$ of $M_{0}$. The uncertainty in $M_{r \theta}$ and $M_{r \phi}$ can result in significant uncertainty in the corresponding fault plane solutions.

The isotropic component of the moment tensor is poorly resolved by most data, with $M_{r r}$ appearing to be particularly poorly resolved from surface waves (Dufumier \& Rivera 1997). Consequently, this part of the moment tensor is usually constrained in moment tensor inversions.

Although tectonic earthquakes are generally assumed to be pure slip on a fault, reported moment tensors often display significant non-DC behaviour. This can happen for a variety of reasons: if the fault plane is curved, if multiple faults of differing orientation (and/or slip direction) are activated (near-)simultaneously, if there is a change in the direction of rupture during the event, if significant axial strain is present (for example in volcanic environments), or if near-source anisotropy is present (see e.g. Knopoff \& Randall 1970; Ekström 1994; Shuler et al. 2013). If complexity of the source itself is the cause of the non-DC parts of the moment tensor, this does not pose a problem in tomography. However, results by Hejrani et al. (2017) indicate that these are often the result of un-accounted for 3-D structure. In their analysis, sources become significantly more DC-like when a detailed 3-D model is used in conjunction with full 3-D wavefield simulations. Indeed, the majority of inverted GCMT moment tensors display significant non-DC behaviour - even those believed to be simple, tectonic events - indicating that this may be a relevant concern.

The scalar moment of an earthquake can be affected by crustal structure (e.g. Patton \& Randall 2002; Abercrombie \& Ekström 2001), and there may be trade-offs between scalar moment and source duration (Vallée et al. 2011). However, if only phase information is used in the tomography, errors in the magnitude play no role.

\section{METHODS}

In the analyses presented in the sections below, we make use of 3-D elastic wave propagation simulations, performed using the spectral element software package Salvus (Afanasiev et al. 2019). Spectral element methods are a higher-order variant of classical finite element methods, and are capable of dealing with irregular grids containing complex geometries and diverse forms of bound- 
ary conditions, while also being highly scalable on modern parallelised computing architectures (e.g. Seriani \& Priolo 1994; Komatitsch \& Tromp 1999; Afanasiev et al. 2019). The elastic wave equation is solved numerically where the discretisation in space and time is determined by the model geometry and the frequency content of the signal. The spatial density of the grid depends on the shortest wavelength, while the timestep is dictated by the propagation velocity of the signal and the spatial discretisation. This means that the computational cost of a simulation scales with approximately the fourth power of the highest frequency in the desired signal (Fichtner 2010).

Tomography is based on a comparison of observations with their synthetically computed equivalents. For the most straightforward case of traveltime tomography, this comparison consists of a differential traveltime which is computed by subtracting a model prediction from an observed traveltime. Such a traveltime difference can also be computed from a comparison of (observed and synthetic) seismograms using cross-correlation:

$\Delta \tau\left(u, u_{\mathrm{obs}}\right)=\underset{t}{\operatorname{argmax}} \int_{t} u\left(t^{\prime}\right) u_{\mathrm{obs}}\left(t+t^{\prime}\right) \mathrm{d} t^{\prime}$

where $\Delta \tau$ is the travel time shift for the synthetic and observed seismograms $u$ and $u_{\mathrm{obs}}$, respectively. Many early and current waveform tomography studies make use of this measurement, because it is stable as long as both seismograms are reasonably similar and the time-shift does not exceed a half-cycle (Luo \& Schuster 1991).

Another common measure is the time-frequency phase misfit formulation as developed by Fichtner et al. (2008). Here the measurement $\Delta \phi(t, \omega)=\phi(t, \omega)-\phi_{\text {obs. }}(t, \omega)$ is made on the time-frequency representation of the seismograms, which are based on the Fourier transform $\tilde{u}$ of a trace $u$ over a sliding time window $h\left(t^{\prime}-t\right)$ :

$$
\begin{aligned}
\tilde{u}(t, \omega) & =\frac{1}{\sqrt{2 \pi}} \int_{\infty}^{\infty} u h^{*}\left(t^{\prime}-t\right) e^{-i \omega t^{\prime}} \mathrm{d} t^{\prime} \\
& =|\tilde{u}(t, \omega)| e^{i \phi(t, \omega)}
\end{aligned}
$$

As for the traveltime cross-correlation, the measurement $\Delta \phi$ is only physically meaningful between $-\pi$ and $\pi$, i.e. when the signals are out of phase by less than half a period. Because of the time-frequency formulation, even small wavefield perturbations can in principle lead to strong phase variations, such that discontinuities are likely to occur, and the measurement in prac- 
tice needs to be stabilised by a weighting function $W_{p}$. Following Fichtner et al. (2008), we use $W_{p}=\frac{\log \left(1+\left|\tilde{u}_{\mathrm{obs}} .\right|\right)}{\max \left(\log \left(1+\left|\tilde{u}_{\mathrm{obs} .}\right|\right)\right)}$ and use the Gabor transform for the window function $h$. To create a single measurement $\Delta \phi$, we here take the maximum of the windowed measurement in time-frequency space:

$\Delta \phi=\max \left(W_{p} \Delta \phi(t, \omega)\right)$

All these measurements can be used as the basis for a misfit functional in a tomographic context. In our discussion we will limit ourselves to those described, although numerous others exist (e.g. Bozdă̆ et al. 2011; Yuan et al. 2015).

\section{PERTURBATION ANALYSIS}

In our first application, we present a perturbation analysis of the effect of errors in earthquake source parameters on the cross-correlation travel-time as measured between perturbed and unperturbed data (Equation 5). For most examples, we use a strike-slip event (a pure DC) located at a reference depth of $10 \mathrm{~km}$. Wave propagation is simulated for the isotropic version of the 1-D Earth model PREM (Dziewoński \& Anderson 1981) for 600 s. We record synthetic velocity seismograms at the surface using a rectangular grid of 405 stations. We select separate windows for body and surface waves on each seismogram (see Supplementary Material section S2) and analyse the results in a frequency band of 28-150 s - a common frequency range in waveform tomography at continental scales. Both spatial and moment tensor perturbations are investigated. Spatial perturbations consist of a $5 \mathrm{~km}$ shift in the east $(+\phi)$ and vertical (+depth) directions. Perturbations to the moment tensor are investigated in terms of dip changes in the fault plane solution. We do this for both a strike-slip fault and a thrust fault, where each are perturbed by $5^{\circ}$. These changes in dip translate to perturbations to the moment tensor components $M_{r \theta}$ and $M_{r \phi}$, which are the components that are typically most poorly constrained (Section 3.4). The perturbations are small compared to realistically expected values (Section 3) in order to minimise the effect of non-linearity while still giving observable results.

We repeat these experiments for an event at a reference depth of $100 \mathrm{~km}$. Although most of 
our results are presented for a strike-slip event, our findings are transferable to other parameters. The model domain we use mimics the central/eastern Mediterranean setup of Blom et al. (2020), but it should be noted that all results presented here are purely synthetic.

In the results presented below, the cross-correlation traveltime shift is always computed such that a positive $\Delta \tau$ means that the signal from the perturbed source arrives earlier than that of the unperturbed source. $\Delta \tau$ is computed automatically for all windows, but becomes somewhat meaningless in locations where the traces differ significantly. This is typically the case at the nodal planes, where amplitudes are small and (numerical) noise can easily distort the measured timeshift. As a result, anomalous values are measured at some of the stations.

Equivalent results for time-frequency phase shifts (Equation 7) are included in the Supplementary Material, Section S4.2.

\subsection{Horizontal and depth perturbations}

Figures 2, 3 and 4 demonstrate the effect of a $5 \mathrm{~km}$ spatial perturbation in source position in the horizontal and depth directions. As expected, a horizontal perturbation results in an azimuthal pattern of time shifts, whereas a depth perturbation results in a radial pattern. The effect of a horizontal perturbation is much more pronounced than the effect of a depth perturbation of the same size, resulting in time shifts between the reference and perturbed events on the order of $>1 \mathrm{~s}$, compared to $<0.5 \mathrm{~s}$ in the case of a depth change (Figure 4). This reflects the reduced sensitivity of long-period signals to earthquake depth.

For a horizontal shift, the effect is much more pronounced for surface waves than for body waves (Figures 2 and 4a), an effect that can likely be attributed to the lower velocities near the surface. The effect of a depth perturbation on surface waves is predominantly on their amplitude; as a result the recorded time shifts are near-zero throughout the entire domain (Figures 3 and $4 b$ ). For body waves, the effect of a depth change is also small, but much clearer (Figure 4b). Outside of a small near-source area, all recorded time shifts are negative, i.e. the perturbed event results in later arrivals. This is contrary to expectation, as the perturbed event is deeper, but probably results from interference of direct and depth phases. The time shift caused by a depth perturbation is quite 
[ht]
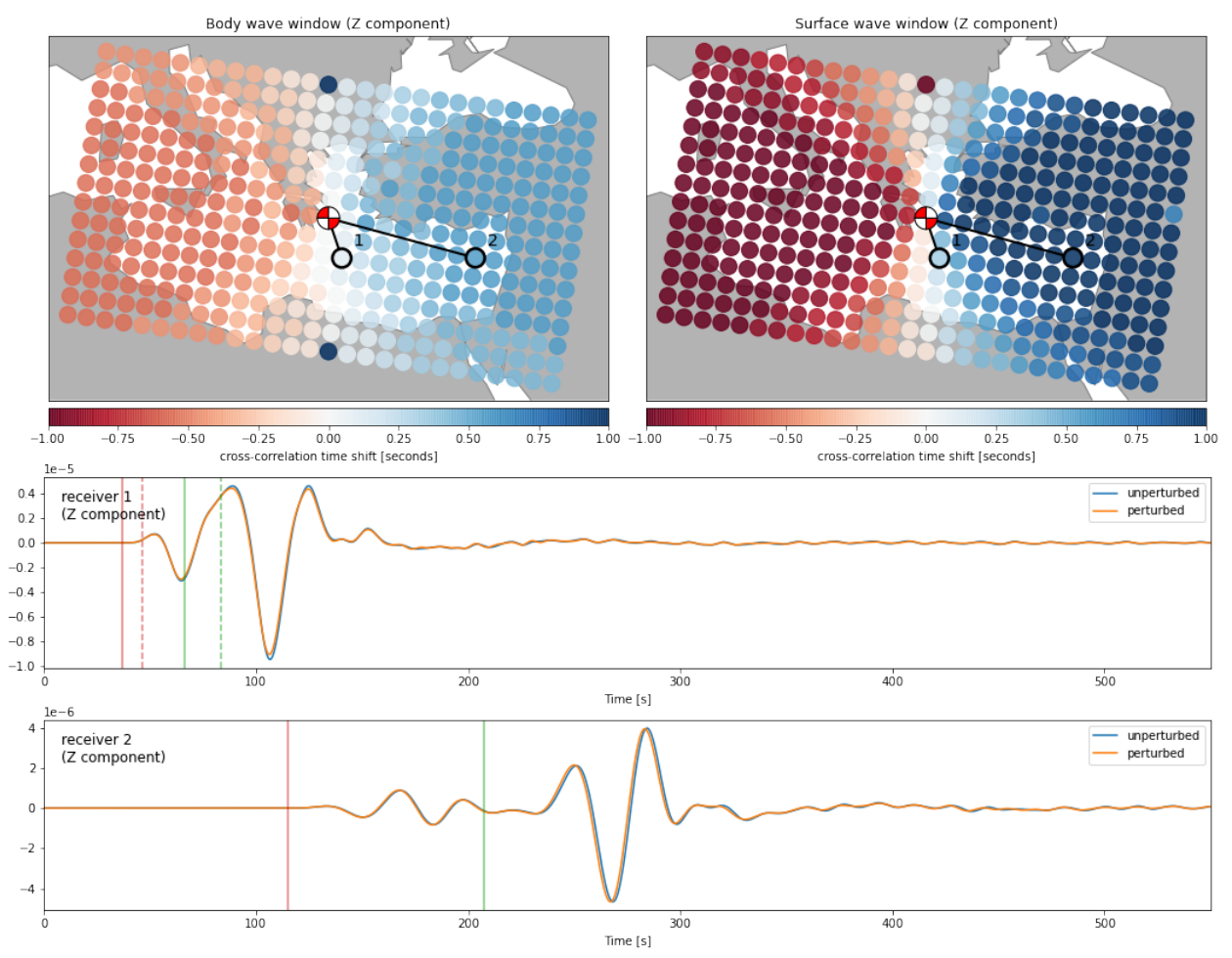

Figure 2. The effect of an eastward shift of the source by $5 \mathrm{~km}$ on seismograms in the frequency band of 28-150 s. This results in a $1 \mathrm{~s}$ time shift for surface waves, and less for body waves. Results are shown for the vertical component. Vertical lines indicate TauP (Crotwell et al. 1999) predicted arrival times for P-waves (red) and S-waves (green), with upgoing phases (depth phases) dashed. Large timeshifts may be recorded at the nodal planes as a result of the small amplitudes interacting with numerical noise.

invariant as a function of distance and without proper station sampling near the source it can easily be mistaken for a uniform shift in origin time. This highlights the well-known trade-off between source depth and origin time.

These results are essentially the same for an event a at greater depth of $100 \mathrm{~km}$ (Figure 4, and see also the Supplementary Material Section S4.1). The horizontal timeshift measurements are virtually unaffected (Figure 4a,b) while the measured timeshifts for body waves as a result of a depth shift increase at large distances (Figure 4c,d).

\subsection{Errors in the moment tensor}

The effect of changes in the moment tensor are generally smaller and consequently typically most noticeable near the nodal planes, where amplitudes are usually small (see the radiation patterns 
[ht]
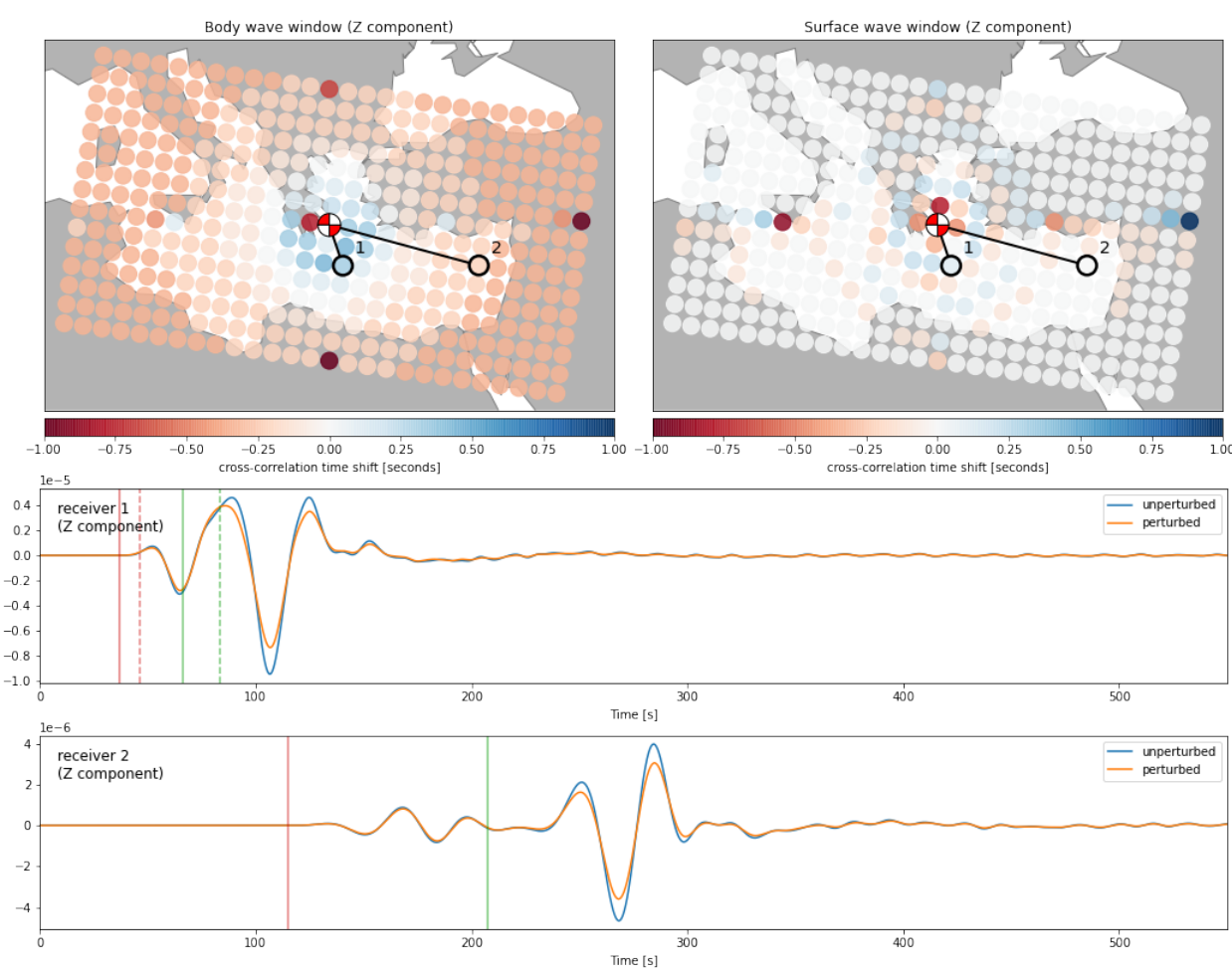

Figure 3. The effect of a $5 \mathrm{~km}$ increase in source depth on seismograms in the frequency band of $28-150 \mathrm{~s}$. The effect is much smaller than the effect of an equally large horizontal shift: for the body wave windows, time shifts of less than $0.5 \mathrm{~s}$ are measured, while the values for surface waves are near-zero (see also Figure 4). Results are shown for the vertical component (Z). (See Figure 2 caption for a full explanation of the figure.)

in Supplementary Figure S2). Figure 5 shows the effect of a $5^{\circ}$ change in dip on the N-S striking fault plane of a strike-slip fault (from $90^{\circ}$ to $85^{\circ} \mathrm{E}$ ). This is the result of a small $M_{r \theta}$ perturbation of magnitude $\sim 0.09 \cdot M_{0}$. Near the N-S striking nodal plane, this effect is visible as a substantial apparent phase shift, but this effect drops off quickly further away from the nodal plane. It is interesting to note that the effect on the body wave window and the effect on the surface wave window are of opposite sign.

In comparison, the effect of such a change in dip is much larger for a $45^{\circ}$ thrust fault (from $45^{\circ}$ E to $40^{\circ} \mathrm{E}$ ), and visible across the entire domain (Figure 6 ). In this case, to create a $5^{\circ}$ change in dip, a $M_{r \phi}$ perturbation of magnitude $-0.18 \cdot M_{0}$ is introduced - approximately twice as large as for the vertical fault plane but still on the small end of the reported typical errors for these components (15-50\%, Valentine \& Trampert 2012). A strong azimuthal pattern of positive and negative time 
[ht]

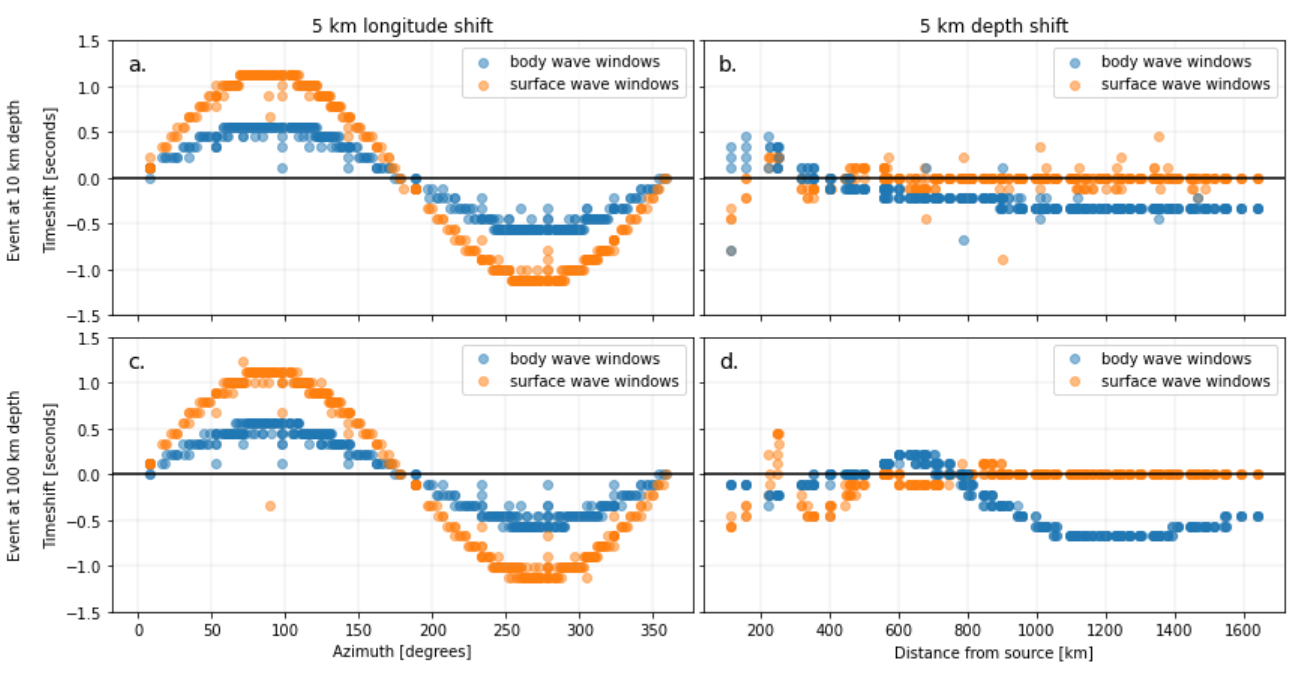

Figure 4. The effect of horizontal and depth shifts of the source on measured cross-correlation time shift. (a) The effect of a horizontal shift in source position of $5 \mathrm{~km}$ (in the longitude direction) as a function of azimuth for an event at a reference depth of $10 \mathrm{~km}$. A clear azimuthal pattern is visible for both body and surface waves. (b) The effect of a $5 \mathrm{~km}$ depth shift as a function of distance from the earthquake for an event at a reference depth of $10 \mathrm{~km}$. Outside of a small area near the source, this effect is nearly uniform. (c) Same as (a), but for an event at $100 \mathrm{~km}$ depth. (d) Same as (b), but for an event at $100 \mathrm{~km}$ depth.

shifts is visible, akin to a horizontal position shift, with again the body and surface wave windows showing timeshifts of opposite sign. This highlights a potential trade-off between the horizontal position of a thrust fault and its dip - consider also Figure 1 .

\section{SYNTHETIC INVERSIONS}

Errors in source parameters will influence the recovered model in a tomographic inversion, but the degree of impact depends on the type and size of the perturbation and the station coverage. We demonstrate this effect for a few of the perturbations from Section 5 using two different station setups: one with the full grid of receivers as described in Section 5 (Figure 7a), and one using only the outer rows of stations (Figure 7b). While neither of these setups are particularly realistic, they demonstrate how station coverage affects the imprint of source errors on tomographic results. Both the target and initial model are the isotropic version of PREM in these inversions; all inverted structure is thus purely a result of the erroneous source. We invert for $v_{\mathrm{p}}, v_{\mathrm{s}}$ and density. We show results for a horizontal shift, a depth shift and a change in dip of the fault plane, using the same 
[ht]
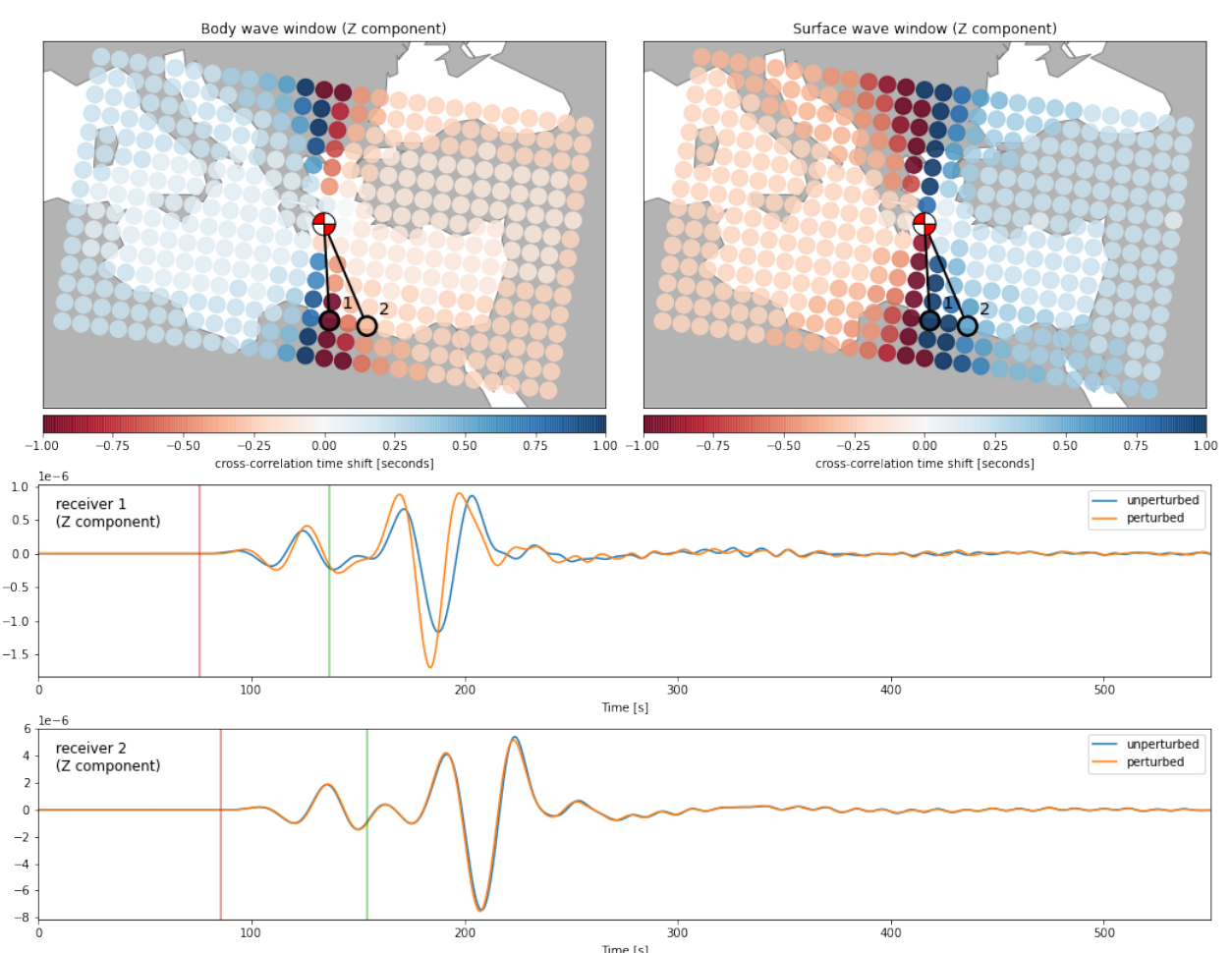

Figure 5. The effect of a $5^{\circ}$ change in the dip of the fault plane striking N-S $\left(90^{\circ}\right.$ to $85^{\circ} \mathrm{E}$ ), on seismograms in the frequency band of $28-150 \mathrm{~s}$. This corresponds to a $M_{r \theta}$ perturbation of $\sim 0.09 \cdot M_{0}$. In general, the effect is quite small, apart from the azimuthal directions that align with the nodal plane that has been altered - however, note here the smaller amplitude of the seismograms recorded at receiver 1 compared to receiver 2 - see Supplementary Material section S3.

setups and events as used in the perturbation analysis of Section 5 (Figures 2-6). Results are summarised in Table 1.

For each of the experiments, five iterations are run where the target data are generated based on the perturbed event, while the inversion is conducted assuming the reference event is positioned in the centre of the domain (as drawn in Figure 7). The iterative non-linear inversion scheme we adopt is based on the trust region approach that makes use of the L-BFGS approximation of the Hessian, following van Herwaarden et al. (2020). Data consist of velocity seismograms bandpass filtered between 28-150 s. As in Section 5, misfits between observed and synthetic data are computed for two windows within each trace: one centred around the body wave arrivals, and one centred around the surface wave arrival. Here, however, we use the time-frequency phase misfit of Fichtner et al. (2008). Alongside information about timeshifts, this misfit also takes into account 

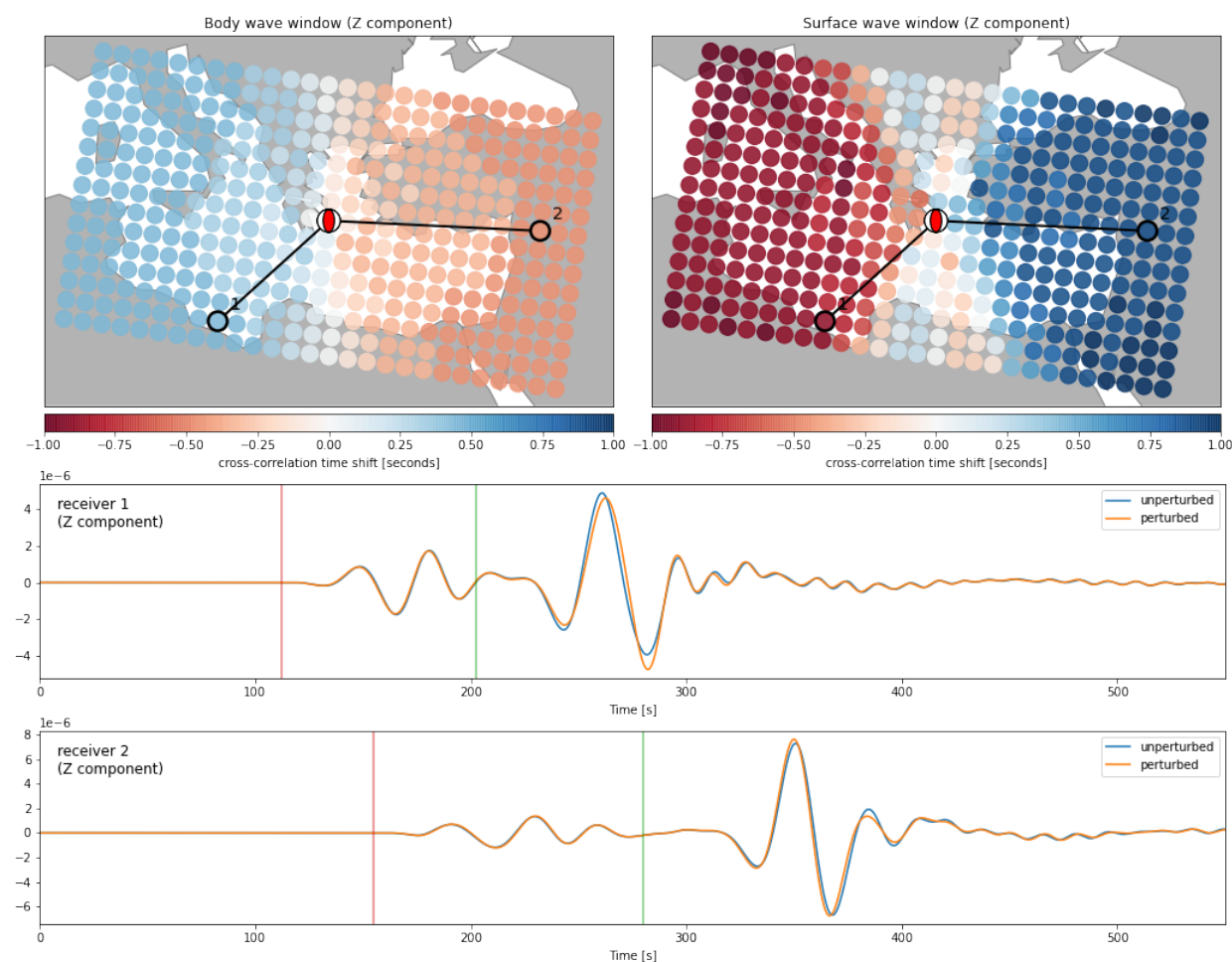

Figure 6. The effect of a $5^{\circ}$ change in dip of the fault plane of a thrust fault (from $45^{\circ} \mathrm{E}$ to $40^{\circ} \mathrm{E}$ ), on seismograms in the frequency band of 28-150 s. In this case, this corresponds to a $M_{r \phi}$ perturbation of $\sim-0.18 \cdot M_{0}$. Because the radiation pattern of the original and perturbed moment tensor align, the effect is visible across the entire domain.

the differing shapes of waveforms, and thus is a powerful tool in tomography for making optimal use of the information supplied in "full" waveforms. Stations with anomalously large misfits are removed from the inversion in order to avoid the inversion being dominated by a single or a few stations - as could be the case near the nodal planes where amplitudes are small (see Section 5). Finally, in order to stabilise the inversion and avoid artefacts resulting from numerical noise, limited smoothing is applied to the model updates, with an isotropic smoothing length of $50 \mathrm{~km}$.

The resulting models are shown in Figure 7 as relative $v_{\mathrm{s}}$ perturbations. For the horizontal shift (Figure $7 \mathrm{c}, \mathrm{d}$ ), the artefacts that form in the inversions are slow in the western part of the domain and fast in the east; a result of the fact that the target data are generated from an event shifted to the east by $5 \mathrm{~km}$. The shape and the amplitude of the artefacts are very different for the two different station setups. The source imprint for the dense station grid (Figure 7c) is concentrated around the source in an area that is about $400 \mathrm{~km}$ in diameter. This corresponds to about four 


\begin{tabular}{|c|c|c|c|c|}
\hline Source parameter & Perturbation & Station configuration & Artefact amplitude & Artefact extent \\
\hline \multirow{2}{*}{ horizontal position } & \multirow{2}{*}{$5 \mathrm{~km}$} & all & $1 \%$ & $400 \mathrm{~km}$ \\
\hline & & only distant & $0.8 \%$ & $>2000 \mathrm{~km}$ \\
\hline \multirow{3}{*}{ depth position } & \multirow{3}{*}{$5 \mathrm{~km}$} & all & $1.5 \%$ & $100 \mathrm{~km}$ \\
\hline & & no near-source & $0.5 \%$ & $100 \mathrm{~km}$ \\
\hline & & only distant & $<0.1 \%$ & - \\
\hline \multirow{4}{*}{ strike-slip dip } & \multirow{4}{*}{$5^{\circ}$} & all & $0.5 \%$ & $400 \mathrm{~km}$ \\
\hline & & no near-source & $0.5 \%$ & $400 \mathrm{~km}$ \\
\hline & & no near-source/nodal-plane & $0.2 \%$ & $500 \mathrm{~km}$ \\
\hline & & only distant & $<0.1 \%$ & - \\
\hline \multirow{2}{*}{ thrust dip } & \multirow{2}{*}{$5^{\circ}$} & all & $0.8 \%$ & $400 \mathrm{~km}$ \\
\hline & & only distant & $<0.1 \%$ & - \\
\hline horizontal position & $20 \mathrm{~km}$ & all & $>5 \%$ & $800 \mathrm{~km}$ \\
\hline horizontal position & $5 \mathrm{~km}$ (at $100 \mathrm{~km}$ depth) & all & $0.8 \%$ & $>1000 \mathrm{~km}$ \\
\hline depth position & $5 \mathrm{~km}$ (at $100 \mathrm{~km}$ depth) & all & $1 \%$ & $500 \mathrm{~km}$ \\
\hline thrust dip & $5^{\circ}$ & all; only surface waves & $1.5 \%$ & $800 \mathrm{~km}$ \\
\hline
\end{tabular}

Table 1. Overview of typical source errors and their effect on tomography. Results for the upper set of tests are visualised in Figures 7 and 8. Additional results (below the double horizontal line) are shown in Supplementary Figures S9-S11.

wavelengths of the dominant signal within the period band used. The artefact is limited in depth to the upper $\sim 50 \mathrm{~km}$. For the inversion using only distant stations (Figure $7 \mathrm{~d}$ ), the source imprint stretches horizontally over more than $1000 \mathrm{~km}$ in either direction, and is strongest on the E-W line (i.e. the direction of perturbation). It is of somewhat smaller amplitude than in the dense setup, but the depth extent is similar. The inversion using only distant stations is much better able to accommodate the measured discrepancy between observed and synthetic data, resulting in a misfit reduction of $62 \%$, compared to $36 \%$ for the dense grid.

The effect of a $5 \mathrm{~km}$ increase in depth is much more limited, as shown in Figure 7e and $\mathrm{f}$. For the dense station grid, the source imprint is strong but very localised, remaining within a wavelength of the earthquake (Figure 7e), while the source error has hardly any effect on the 


\section{6}

N.A.Blom, N. Rawlinson

recovered model in the inversion where only distant stations are used (Figure 7f). The near-source slow artefact reflects the fact that the true source is deeper than the one used in the inversion.

The effects of moment tensor perturbations are variable. For the strike-slip fault, a $5^{\circ}$ change in the dip of the N-S striking fault (from $90^{\circ}$ to $85^{\circ} \mathrm{E}$ ) has a moderate effect on the recovered model, again displaying a degree-2 pattern of artefacts (Figure $7 \mathrm{~g}$ ). These artefacts are lower in amplitude than the artefacts resulting from a horizontal shift, and relatively limited in extent, although here, too, faint additional artefacts are visible towards the north and south. If only distant stations outside of the neighbourhood of the perturbed nodal plane are used in the inversion, no source effect is visible in the result at all (Figure $7 \mathrm{~h}$ ).

Changing the dip of a thrust fault by $5^{\circ}$ (from $45^{\circ} \mathrm{E}$ to $40^{\circ} \mathrm{E}$ ), in contrast, has a significant effect on the recovered model. For the dense grid, this results in a strong source imprint on the model of a similar degree-2 pattern as the horizontal shift (Figure 7i). Visually, the surface waves of the thrust dip change look similar to those of the eastward horizontal perturbation, and positive time shifts are indeed recorded east of the source and negative time shifts to the west (Figures 2 and 6). The inverted model for the thrust dip change, however, has artefacts of opposite sign near the surface, which reverse in sign at about $20 \mathrm{~km}$ depth. This effect occurs even in an inversion using only surface wave windows (see Supplementary Figure S11), indicating that it is entirely due to subtle deviations in the shape of the surface waveforms. The inversion using only distant stations is hardly affected by the erroneous source, indicating that here, too, the adverse effect on tomography is largely restricted to the near-source area (Figure 7j).

Similar experiments with an event located at a reference depth of $100 \mathrm{~km}$ largely mirror these results (Supplementary Figure S10). However, for the deep source, the effect of a depth shift in particular is visible across a much larger region than for the shallow reference depth, spanning about $400 \mathrm{~km}$ (as opposed to $100 \mathrm{~km}$ ), and also has a larger amplitude. This is also true if only distant stations are used, although the effect of the erroneous depth is still much more limited than in an inversion also using nearby stations. 

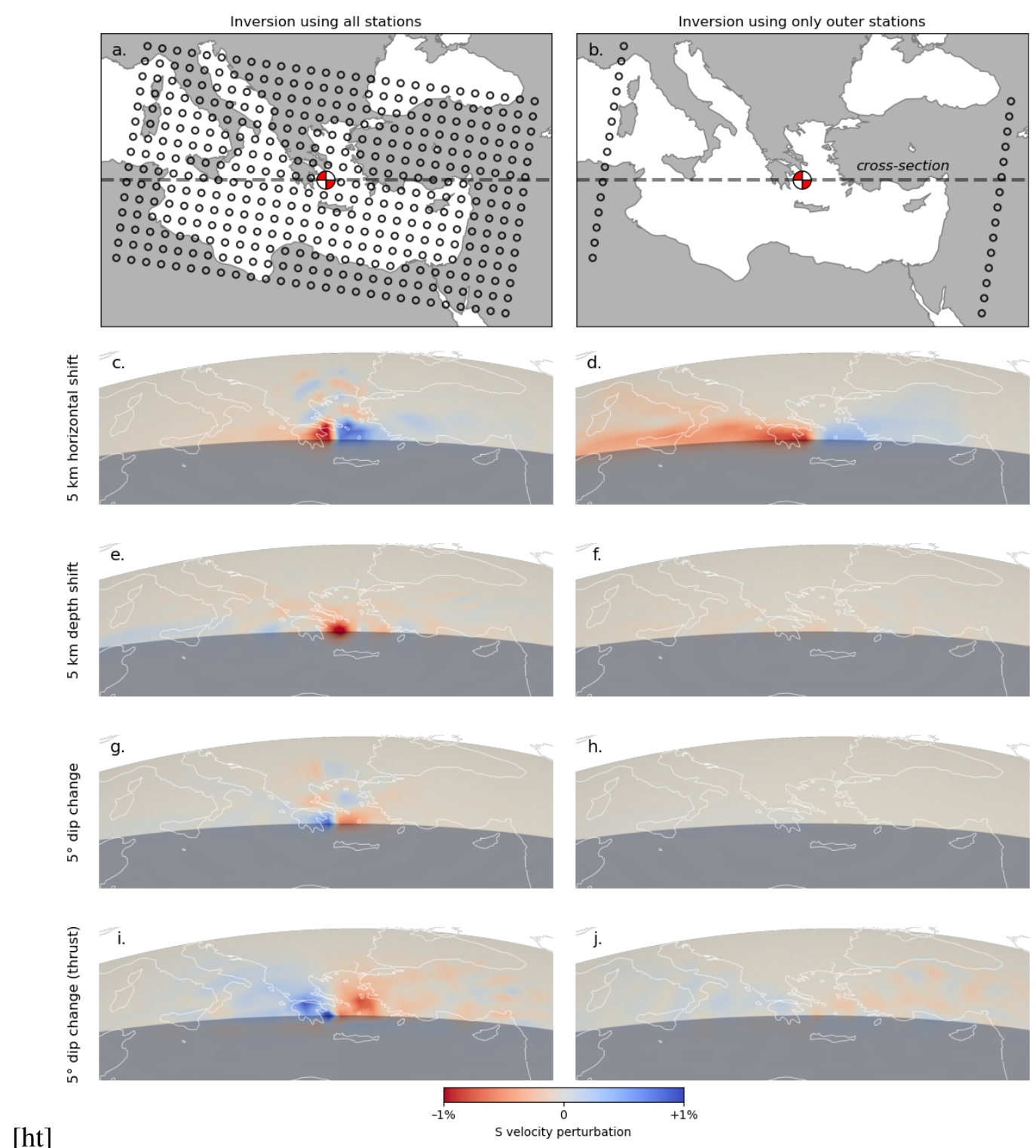

Figure 7. Result of a tomographic inversion where 'observed' data are generated from a source which is perturbed relative to the source used in the inversion, in the same setup as used in the perturbation analysis of Section 5. In the ideal case, the inversion result would be zero perturbation. We demonstrate inversion results for either a full grid of receivers or using only the outer stations, and show the resulting $v_{\mathrm{s}}$ model after 5 iterations as relative perturbations to the starting model. (a) Inversion setup with full grid of receivers. (b) Inversion setup with only outer stations. (c) Inversion result where 'observed' data are generated from an event shifted by $5 \mathrm{~km}$ to the east. Section through the final relative $v_{\mathrm{s}}$ model after 5 iterations using the full grid setup, along the dashed line shown in (a). (d) Section through the final model after 5 iterations using the outer stations setup (horizontal perturbation), along the dashed line shown in (b). (e,f) As c and d, but for a perturbation in the depth direction by $5 \mathrm{~km}$. (g,h) As $\mathrm{c}$ and d, but for a $5^{\circ}$ perturbation of the fault plane of a N-S striking $45^{\circ}$ thrust fault. Model sections were produced using ParaView (Ahrens et al. 2005). 


\section{DISCUSSION}

Significant uncertainties are present in the source parameters of most catalogue events, and have the potential to affect seismic tomography results regardless of the method used. These issues are of particular concern for (adjoint) waveform tomography, where heterogeneities are generally stronger and the inverse problem significantly more non-linear compared to traditional ray-based methods. Below, we discuss our results in more detail and consider several potential mitigation strategies. Although the focus of our discussion is waveform tomography, many of the points are equally valid for other methods that rely on time- or phaseshift measurement.

\subsection{Generalisation of the results}

In order to keep the effect of non-linearity in our results to a minimum, all perturbations that we have analysed above are small compared to the typically expected values. Spatial uncertainties can reach tens of kilometres, whereas our analyses are done using $5 \mathrm{~km}$ perturbations. Therefore, while the amplitudes of the inverted source error artefacts in our toy inversions are small compared to velocity perturbations typically found with waveform tomography, in realistic settings they will be much more significant for each of the parameters considered. Moreover, all inversions were run for five iterations, regardless of whether misfit development had stabilised or not. In a realistic tomography application, source-related artefacts would thus continue to accumulate over possibly tens of further iterations.

To demonstrate the effect of a more realistic perturbation, we have conducted an additional inversion with a horizontal source perturbation of $20 \mathrm{~km}$. In this case, the resulting anomalies are on the order of $5 \%$ (instead of 1\%), and extend over a larger area (Supplementary Figure S9). In general, extrapolation of our results to more realistic error sizes can be undertaken, but with some caution. Because we use a phase-based measurement in our misfit, none of the tomographic effects have a linear relationship with the source perturbation. However, as long as the source perturbation results in seismograms that remain well within a half-cycle, a more or less linear effect can likely be assumed. All extrapolation obviously depends on the signal frequency and local wavespeed and becomes more complicated when 3-D structure is present. 
It is also important to consider how these results extrapolate to events at greater depth. Crustal earthquakes form a large proportion of earthquakes used in waveform tomography, and $10 \mathrm{~km}$ is a very typical depth for these. However, in subduction zones in particular, much deeper events are likely to be found and used. In our experiments, the resulting anomalies are of a significantly larger extent for an event at a reference depth of $100 \mathrm{~km}$ (Supplementary Figure S10). This is likely the result of the fact that there is more space to accommodate the information in the phaseshift measurements.

A single event can generate non-negligible artefacts in an inversion. However, as discussed in Section 3, systematic errors for a region are very common, for example in areas of strong lateral heterogeneity such as subduction zones. If tomography is conducted using a suite of events such as those in Figure S1, the individual artefacts will add up, resulting in the creation of significant erroneous structure.

Our results are based on measurements of time and phase shifts. The fact that horizontal spatial errors result in more significant artefacts than depth errors is partially due to this. If observations of amplitudes form the basis of the tomographic inversion, it is likely that the effect on the resulting model of (for example) a depth error will be much more significant. However, time and phase shifts are very common types of measurements used in seismic tomography, meaning that our results have reasonably broad applicability.

\subsection{Joint source-structure inversions}

Various authors (e.g. Pavlis \& Booker 1980; Valentine \& Woodhouse 2010) have presented methods to include source parameters into the tomographic inversion, and in some sub-disciplines of seismic tomography, it is indeed standard practice to perform joint source-structural inversions (e.g. in local earthquake tomography). Our work demonstrates that the effects of not doing this are important and can extend significantly beyond the source region. Indeed in waveform tomography such practices would ideally be adopted, but this is hampered by several factors, as discussed below.

A problem that all joint inversions struggle with is differing levels of non-linearity. This prob- 


\section{0}

N.A.Blom, N. Rawlinson

lem is particularly acute if a subset of parameters has a linear forward relationship while others are related to the data in a non-linear way. This is the case for the CMT inversions, which is one of the reasons for the relative complexity of the GCMT algorithm (Section 2.2; Ekström et al. 2012). Another example is the joint inversion of seismic and gravity data for density, where gravity data is linearly related to density in a very non-unique way, while the relationship between seismic data and density is non-linear and indirect (see e.g. Tondi et al. 2009; Maceira \& Ammon 2009; Simmons et al. 2010; Moulik \& Ekström 2016; Blom et al. 2017). The problem also occurs if different levels of non-linearity are present, as is the case in traveltime tomography when jointly inverting for sources: here the velocity inversion is also much less non-linear than the source location problem (e.g. Pilia et al. 2013). Poor coverage (especially azimuthally) can easily lead to unlikely source position shifts. Common mitigation efforts centre on finding suitable damping parameters, empirical scaling relationships and the inclusion of a priori information. All mitigation measures (including the design of the inversion algorithm and the tuning of damping parameters) make the inversion more strongly subjective, and its results less reproducible.

In waveform tomography, an additional concern is computational cost. To compute the gradient with respect to velocity and density, two simulations are necessary per event. In theory, for each source parameter two additional simulations would be needed in order to construct their gradients (Liu et al. 2004). Jointly inverting for structure and sources would thus result in an order of magnitude increase in computational cost, which quickly becomes intractable in light of the non-linearity of the source inversion problem. Significant efforts have been made in recent years to develop efficient source inversion algorithms for waveform tomography, with in particular the concept of spatial reciprocity (the Green's function between a source and receiver being equal in both directions) proving to be an important avenue of inquiry (e.g. Zhao et al. 2006; Hejrani et al. 2017; Fichtner \& Simute 2018). By computing wavefields per receiver instead of per source, the same receiver wavefields can be used for the inversion of all sources. This results in computational gains when inversions for many sources can be carried out based on relatively few receivers, but is also helpful to explore the entire model space of potential source parameters and their tradeoffs (Fichtner \& Simute 2018). If based on a high-quality Earth model, such precomputed Green's 


\begin{tabular}{lll} 
Source parameter & Significance & Mitigation \\
\hline horizontal position & significant & difficult; compare to ground-truth data or discard event. \\
depth position & localised & remove source area from gradient or exclude near-source stations \\
strike-slip dip & moderate & exclude stations with small amplitude traces, in particular near nodal planes
\end{tabular}

Table 2. Significance and mitigation of typical source errors.

functions can be a powerful tool for the purpose of routine source inversions, and are helpful for extremely quick source characterisation in the context of earthquake early warning systems.

In the context of tomography, however, the Earth model changes with each iteration and Green's functions consequently need to be recomputed for each model. More work thus needs to be done to develop efficient methods to allow joint source-structual waveform tomography. Meanwhile, occasional source inversions such as that carried out by e.g. Fichtner et al. (2013) can be considered - a method that can potentially be optimised by making use of reciprocity using a selection of 'gold standard' stations distributed optimally over the model domain in order to maximise (azimuthal) coverage for all earthquakes involved.

\subsection{Mitigation strategies}

Instead of including source parameters in the inverse problem formulation, we can also assess how the adverse effects of errors in them can be minimised by considering which measurements are sensitive to them. Our results present some handholds, and in the following, we discuss some possible mitigation strategies that are relatively easy to implement. These are summarised in Table 2.

The effect of source error types that we have considered varies significantly in extent and amplitude. With the notable exception of a horizontal position error, it tends fall away almost entirely if only distant stations are used in the inversion (Figure 7). A worthwhile mitigation measure thus appears to be the removal of a source imprint area from the constructed event gradient. The size of this area depends on the frequency content of the measurements, the event depth, and the velocity structure around the event, but our results indicate that the radius needs to be at least four wavelengths of the dominant signal for shallow events, and may need to increase for deeper events 


\section{2}

N.A.Blom, N. Rawlinson

(see Supplementary Figure S10). This method, whilst removing artefacts resulting from source errors, also removes potentially valuable information on the source region altogether. In a setup where many events originate from the same area, this may result in limited information being gained about this region. This may be problematic when one is interested in e.g. subduction zone structure. An alternative mitigation measure may be to exclude all near-source stations prior to inversion. This strategy is also helpful to mitigate the effects of finite source rupture effects. As such, it is a procedure often followed in waveform tomography, which is for example included in the automated window picking algorithm of the LASIF waveform inversion management toolbox (Krischer et al. 2015; Thrastarson et al. 2021). It should be noted, however, that depending on the frequency content and event depth, the area over which this needs to happen can also get quite large, with the consequent exclusion of a large proportion of stations available for an event (Figure $8)$.

The effects of moment tensor errors tend to become most apparent in areas where amplitudes are low. A case in point is a dip perturbation on a strike-slip fault, where the largest effects are visible along the strike of the perturbed fault plane (see Figure 5). A strategy to avoid source artefacts may thus be to discard any measurement based on parts of the seismogram that are relatively low-amplitude. Supplementary Figure S2 shows radiation patterns highlighting such areas for the different windows and different components considered here. Such measurements may already be excluded because of the presence of noise, but for high-quality stations or large-magnitude events, the signal-to-noise ratio can be excellent even at low amplitude, which could result in the inclusion of a measurement that contributes to the formation of source artefacts. Analysing wavefield amplitude for each source-model combination for all measurements comes at no significant additional computational cost, and can thus be implemented easily. An alternative may be to investigate measurements that have anomalously large misfits, such as the ones discussed in Section 5. Excluding, say, the largest $1 \%$ of misfit measurements may result in stabilisation of the resulting event gradient, and a consequent exclusion of source error artefacts. This strategy may additionally be useful to weed out stations which themselves have problems. What must be kept in mind here is that 'typical' misfit values tend to vary between the horizontal and vertical components. 
We demonstrate the effect of such mitigation strategies in Figure 8. For a depth perturbation, the removal of all receivers within a $\sim 400 \mathrm{~km}$ radius results in a marked decrease of the artefact amplitude, although it does not entirely disappear. This same measure has no discernible effect on the imaged artefacts for a strike-slip dip perturbation. Here, the artefacts only reduce in amplitude if all stations on the N-S striking nodal plane are also removed, i.e. in the area where the largest timeshifts are recorded (Figure 5). Using only distant stations away from this nodal plane area completely removes the artefacts.

\subsection{Mitigating epicentral errors}

The most problematic type of source error is a horizontal location error, which of all perturbations considered has the largest and most far-reaching effect, regardless of whether near or only distant stations are used. This means that none of the mitigation strategies discussed above are helpful to avoid these errors, while even for perturbations to the source that are moderate compared to typical values, the resulting artefacts quickly become similar in amplitude to 'real' velocity structure.

A first step may be to scan the data set for possibly problematic events. One method to identify these is to consider the misfits they produce. The average misfit per measurement tends to be relatively uniform across events for a given model (see the Supplementary Material of Blom et al. 2020), so total event misfit should scale fairly linearly with the number of measurements made. If one event is a clear outlier of the trend, it may be an indication of a source error that is affecting misfits across the entire domain, warranting further investigation. This is the case for epicentral errors, but also for depth or origin time errors which have a relatively uniform effect over the entire domain. This is the approach taken in Blom et al. (2020).

For specific events, one can analyse the spatial patterns of phase- and/or timeshifts. As demonstrated in Figure 1, clear azimuthal patterns may be a warning signal, while a uniform advance or delay across the entire domain could indicate an erroneous origin time, or possibly depth (depending on the dominant measurements used). Several examples are included in the Supplementary material of Blom et al. (2020).

While such maps are an excellent diagnostic tool, they do not hold the key to mitigation. A case 

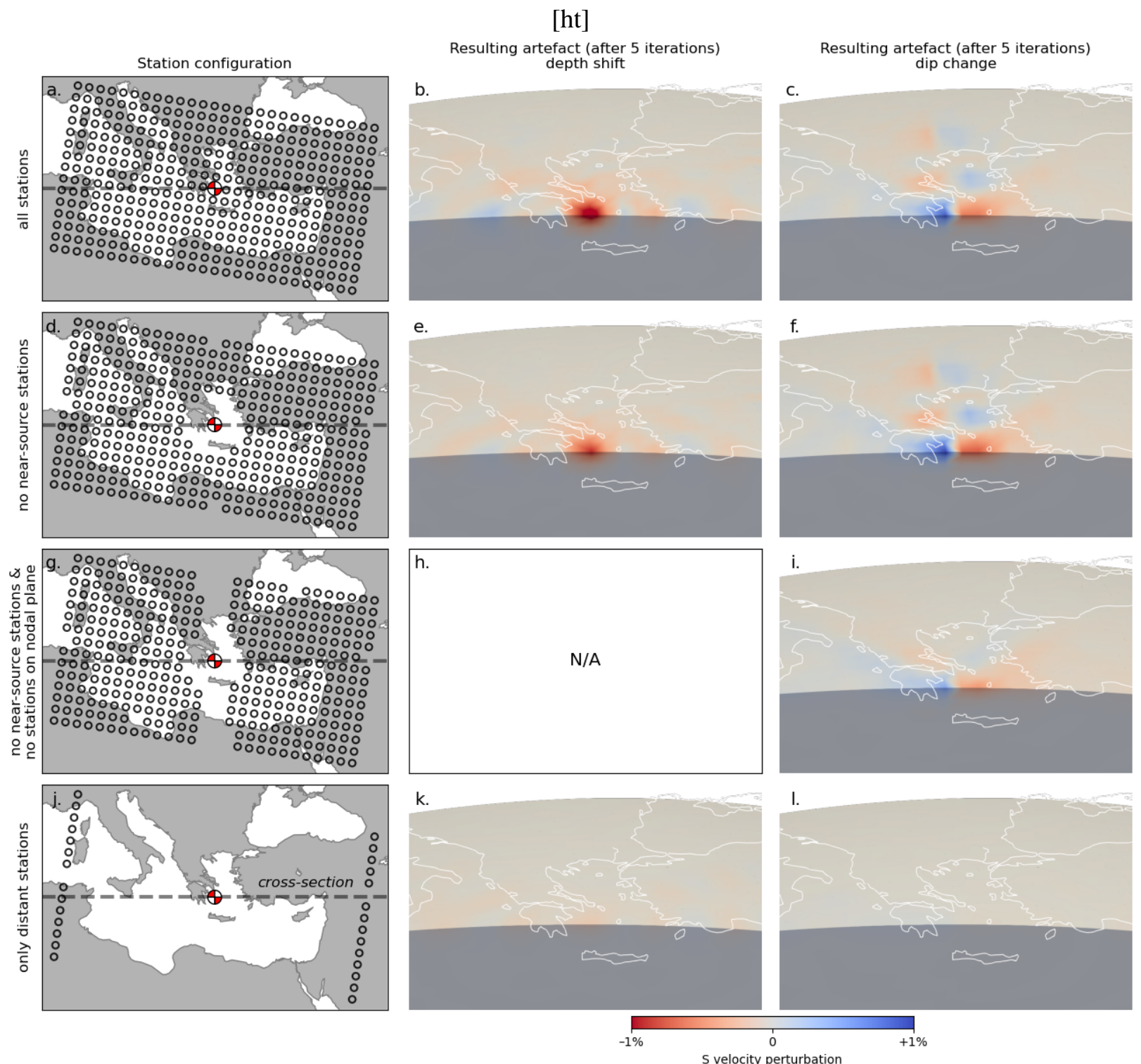

Figure 8. The effect of station inclusion on recovered source artefacts, using the same toy inversion setup as discussed in Section 6. All results are shown after 5 iterations. (a) Inversion setup using all stations. (b) Resulting artefacts for a $5 \mathrm{~km}$ depth perturbation using setup (a). (c) Resulting artefacts for a $5^{\circ}$ dip change on the N-S striking fault plane (from $90^{\circ}$ to $85^{\circ} \mathrm{E}$ ) using setup (a). (d) Inversion setup where receivers within a $\sim 300 \mathrm{~km}$ radius have been removed. (e) Resulting artefacts for a $5 \mathrm{~km}$ depth perturbation using setup (d). (f) Resulting artefacts for a $5^{\circ}$ dip change on the N-S striking fault plane (from $90^{\circ}$ to $85^{\circ} \mathrm{E}$ ) using setup (d). (g) Inversion setup where receivers within a $\sim 300 \mathrm{~km}$ radius have been removed, as well as all stations lying on or near the N-S striking nodal plane. These are the stations with significant misfits for a dip change (see Figure 5). (h) Not shown because irrelevant: Resulting artefacts for a $5 \mathrm{~km}$ depth perturbation using setup (g). (i) Resulting artefacts for a $5^{\circ}$ dip change on the N-S striking fault plane (from $90^{\circ}$ to $85^{\circ} \mathrm{E}$ ) using setup (g). (j) Inversion setup where only distant stations are used. (k) Resulting artefacts for a $5 \mathrm{~km}$ depth perturbation using setup (j). (l) Resulting artefacts for a $5^{\circ}$ dip change on the N-S striking fault plane (from $90^{\circ}$ to $85^{\circ} \mathrm{E}$ ) using setup (j). Model sections were produced using ParaView (Ahrens et al. 2005). 
in point is the example event in Figure 1, where an azimuthal pattern of phase shifts aligns more or less with the strike of the event. While a position shift indeed works to minimise this azimuthal pattern, this would not be the appropriate mitigation measure if the cause of the pattern is a fault plane dip error. If many separate body wave measurements can be made, it may be possible to discern the opposite patterns of phase shifts for body and surface waves, but in general the bulk of measurements in waveform tomography are of surface waves. Moreover, as Creager \& Jordan (1984) remark, "hypocenter mislocation is [...] indistinguishable from any component of lateral heterogeneity whose representation on the residual sphere is dipolar". Mitigation of horizontal errors thus requires the most careful attention.

The most promising mitigation strategy for horizontal errors thus appears to be to compare the reported horizontal position with 'ground truth' data. Such data may be available for shallow events in the form of mapped surface ruptures or InSAR source analyses (e.g. Xu et al. 2015), but may be difficult to obtain for deeper events. These considerations may mean that if an event appears suspicious without reliable additional information about it, the most cautious approach would be to remove it from the dataset.

\section{CONCLUSIONS}

Seismic source parameters reported in catalogues can contain significant errors, which are a result of data errors, errors in how synthetic data are computed and errors in the assumptions made in the source inversion. For tomography, the most significant cause of source errors is probably the Earth model used in the source inversion, in particular because this is updated during inversion. However, data errors can be significant and suboptimal coverage can exacerbate the effect of any error type. Typical position errors can easily reach tens of $\mathrm{kms}$ and errors in the moment tensor components can reach $50 \%$ of the seismic moment, which can translate into fault plane errors of tens of degrees.

We have shown that such errors have a considerable effect on seismic measurements based on time- and phaseshifts. These are the most common types of measurements in seismic tomography and are also typically used in waveform tomography. If unaddressed, they can result in significant 


\section{6}

N.A.Blom, N. Rawlinson

artefacts in the tomographic image extending multiple wavelengths away from the source. The most problematic type of source error is an error in horizonal position, which in tomography results in large-amplitude artefacts that extend over large distances, regardless of whether near or only distant stations are used. Depth errors, in contrast, have a more localised effect, which however becomes more pronounced with event depth. Depth error artefacts disappear if only stations are used which are far away from the source. Errors in the moment tensor components - even in the case of $M_{r \theta}$ and $M_{r \phi}$, which have the smallest effect on the wavefield - can lead to significant measured timeshifts and tomographic artefacts, depending on the event type. In the examples that we have considered, these errors translate into a dip change (for a strike-slip and for a thrust fault), and result in a degree-2 pattern in both phaseshift measurements and inverted artefacts reminiscent of a horizontal spatial error.

Systematic errors in source descriptions are a particular concern, as their effects add up. This is likely to occur in particular in subduction zones (which can result in very large horizontal shifts), or areas with anomalously thick or thin crust (which can result in a strong depth and/or origin time bias).

Although the theoretically ideal tomographic approach is joint inversion of source parameters and structure (Valentine \& Woodhouse 2010), this is challenging in general from an inverse theory perspective and computationally very expensive in the case of waveform tomography. We therefore present a number of relatively straightforward mitigation measures, which together help to minimise the effect of source errors on tomography.

- Removing a near-source area from the event gradient helps to minimise strong, local source artefacts from forming in the inversion, and thus mitigates the effect of several types of source error. The required size of this area depends on event depth, signal frequency, and velocity structure. This is not only helpful for the artefacts we have considered, but also to remove finite source rupture effects. If no near-source stations are present, this may not be necessary. An equivalent method therefore consists of discarding near-source stations, although this might need to be done in a rather large area which leads to the exclusion of a large proportion of possible measurements.

- It may be necessary to discard measurements where the seismograms on which they are based 
have very low amplitude, for example on or near nodal planes. These are the areas most sensitive to moment tensor errors. This depends on the radiation pattern of the given event for the specific measurement window and component.

- Horizontal position errors are not mitigated by the above measures, and require special attention. Event-based misfit and phase shift analyses can serve as diagnostic tools to discover anomalous, erroneous or suspicious events. A suitable correction value may be found if 'ground truth' data is available, which for shallow events can take the form of surface rupture and InSAR analyses. If this cannot be done, the most cautious approach is to discard such an event.

\section{DATA AVAILABILITY}

The data underlying this article will be available in a Zenodo repository, and will additionally be shared on reasonable request to the corresponding author.

\section{Acknowledgments}

Simulations were carried out using Salvus (Afanasiev et al. 2019), which were run on local clusters. All the data analysis in this project was carried out using functionality that ultimately derives from the LASIF package. We thank Lion Krischer, who wrote the original version used in this project, as well as Sölvi Thrastarson and Dirk-Philip van Herwaarden and all others who have contributed to the continued development of this freely available tool. All of this would not be possible without the seismological functionality that has been developed in the ObsPy toolkit. NAB wants to give a special thanks to Saulè Simutė whose knowledge about seismic sources (and willingness to discuss) probably saved her from a great deal of embarrassment. Thanks to Tim Craig for useful discussions on earthquake depth and for sharing his data with us. Also thanks to Sam Wimpenny, Tom Winder and Deborah Wehner for useful input and discussions.

\section{REFERENCES}

Abercrombie, R. E. \& Ekström, G., 2001. Earthquake slip on oceanic transform faults, Nature, 410(6824), 74-77. 
Afanasiev, M., Boehm, C., van Driel, M., Krischer, L., Rietmann, M., May, D. A., Knepley, M. G., \& Fichtner, A., 2019. Modular and flexible spectral-element waveform modelling in two and three dimensions, Geophysical Journal International, 216(3), 1675-1692.

Ahrens, J., Geveci, B., \& Law, C., 2005. Paraview: An end-user tool for large data visualization, The visualization handbook.

Aki, K. \& Lee, W. H. K., 1976. Determination of three-dimensional velocity anomalies under a seismic array using first $\mathrm{P}$ arrival times from local earthquakes - 1. A homogeneous initial model, J. Geophys. Res., 81, 4381-4399.

Amaru, M., 2007. Global travel time tomography with 3-D reference models, Ph.D. thesis, Utrecht University.

Bijwaard, H., Spakman, W., \& Engdahl, E. R., 1998. Closing the gap between regional and global traveltime tomography, J. Geophys. Res., 103, 30055-30078.

Blom, N., Gokhberg, A., \& Fichtner, A., 2020. Seismic waveform tomography of the central and eastern Mediterranean upper mantle, Solid Earth, 11(2), 669-690.

Blom, N. A., Boehm, C., \& Fichtner, A., 2017. Synthetic inversions for density using seismic and gravity data, Geophys. J. Int., 209(2), 1204-1220.

Bozdağ, E., Peter, D., Lefebvre, M., Komatitsch, D., Tromp, J., Hill, J., Podhorszki, N., \& Pugmire, D., 2016. Global adjoint tomography: first-generation model, Geophysical Supplements to the Monthly Notices of the Royal Astronomical Society, 207(3), 1739-1766.

Bozdağ, E., Trampert, J., \& Tromp, J., 2011. Misfit functions for full waveform inversion based on instantaneous phase and envelope measurements, Geophys. J. Int., 185, 845-870.

Craig, T., 2019. Accurate depth determination for moderate-magnitude earthquakes using global teleseismic data, Journal of Geophysical Research: Solid Earth, 124(2), 1759-1780.

Creager, K. C. \& Jordan, T. H., 1984. Slab penetration into the lower mantle, Journal of Geophysical Research: Solid Earth, 89(B5), 3031-3049.

Crotwell, H. P., Owens, T. J., \& Ritsema, J., 1999. The TauP toolkit: Flexible seismic travel-time and ray-path utilities, Seismological Research Letters, 70(2), 154-160.

Dufumier, H. \& Rivera, L., 1997. On the resolution of the isotropic component in moment tensor inversion, Geophysical Journal International, 131(3), 595-606.

Dziewoński, A., Franzen, J., \& Woodhouse, J., 1984. Centroid-moment tensor solutions for JanuaryMarch, 1984, Physics of the earth and planetary interiors, 34(4), 209-219.

Dziewoński, A., Ekström, G., Franzen, J., \& Woodhouse, J., 1987. Centroid-moment tensor solutions for January-March 1986, Physics of the Earth and Planetary Interiors, 45(1), 1 - 10.

Dziewoński, A. M., 1984. Mapping the lower mantle: determination of lateral heterogeneity in p velocity up to degree and order 6, Journal of Geophysical Research: Solid Earth, 89(B7), 5929-5952. 
Dziewoński, A. M. \& Anderson, D. L., 1981. Preliminary reference Earth model, Phys. Earth Planet. Inter, 25, 297-356.

Dziewoński, A. M. \& Woodhouse, J. H., 1983. An experiment in systematic study of global seismicity: Centroid-moment tensor solutions for 201 moderate and large earthquakes of 1981, Journal of Geophysical Research: Solid Earth, 88(B4), 3247-3271.

Dziewoński, A. M., Hager, B. H., \& O’Connell, R. J., 1977. Large-scale heterogeneities in the lower mantle, J. Geophys. Res., 82, 239-255.

Dziewoński, A. M., Chou, T.-A., \& Woodhouse, J. H., 1981. Determination of earthquake source parameters from waveform data for studies of global and regional seismicity, Journal of Geophysical Research: Solid Earth, 86(B4), 2825-2852.

Ekström, G., 1994. Anomalous earthquakes on volcano ring-fault structures, Earth and Planetary Science Letters, 128(3-4), 707-712.

Ekström, G., 2011. A global model of Love and Rayleigh surface wave dispersion and anisotropy, 25-250 s, Geophys. J. Int., 187(3), 1668-1686.

Ekström, G., Morelli, A., Boschi, E., \& Dziewonski, A. M., 1998. Moment tensor analysis of the central italy earthquake sequence of september-october 1997, Geophysical Research Letters, 25(11), 19711974.

Ekström, G., Nettles, M., \& Dziewonski, A. M., 2012. The global CMT project 2004-2010: centroid moment tensors for 13,017 earthquakes, Phys. Earth Planet. Inter., 200-201, 1-9.

Engdahl, E. \& Gubbins, D., 1987. Simultaneous travel time inversion for earthquake location and subduction zone structure in the central Aleutian Islands, Journal of Geophysical Research: Solid Earth, 92(B13), 13855-13862.

Engdahl, E. R., 2006. Application of an improved algorithm to high precision relocation of isc test events, Physics of the Earth and Planetary Interiors, 158(1), 14-18.

Engdahl, E. R., van der Hilst, R., \& Buland, R., 1998. Global teleseismic earthquake relocation with improved travel times and procedures for depth determination, Bulletin of the Seismological Society of America, 88(3), 722-743.

Ferreira, A. M. \& Woodhouse, J. H., 2006. Long-period seismic source inversions using global tomographic models, Geophysical Journal International, 166(3), 1178-1192.

Fichtner, A., 2010. Full Seismic Waveform Modelling and Inversion., Springer, Heidelberg.

Fichtner, A. \& Simutè, S., 2018. Hamiltonian Monte Carlo inversion of seismic sources in complex media, Journal of Geophysical Research: Solid Earth, 123(4), 2984-2999.

Fichtner, A., Kennett, B. L. N., Igel, H., \& Bunge, H.-P., 2008. Theoretical background for continentaland global-scale full-waveform inversion in the time-frequency domain., Geophys. J. Int., 175, 665-685. Fichtner, A., Kennett, B. L. N., Igel, H., \& Bunge, H.-P., 2009. Full seismic waveform tomography for 
upper-mantle structure in the Australasian region using adjoint methods., Geophys. J. Int., 179, 17031725.

Fichtner, A., Trampert, J., Cupillard, P., Saygin, E., Taymaz, T., Capdeville, Y., \& Villasenor, A., 2013. Multi-scale full waveform inversion, Geophys. J. Int., 194, 534-556.

Gilbert, F., 1971. Exitation of normal modes of the earth by earthquake sources, Geophys. J. R. Astron. Soc., 22, 223-226.

Grand, S. P., 2002. Mantle shear-wave tomography and the fate of subducted slabs, Philosophical Transactions of the Royal Society of London. Series A: Mathematical, Physical and Engineering Sciences, 360(1800), 2475-2491.

Hanks, T. C. \& Kanamori, H., 1979. A moment magnitude scale, Journal of Geophysical Research: Solid Earth, 84(B5), 2348-2350.

Hejrani, B., Tkalčić, H., \& Fichtner, A., 2017. Centroid moment tensor catalogue using a 3-d continental scale earth model: Application to earthquakes in papua new guinea and the solomon islands, Journal of Geophysical Research: Solid Earth, 122(7), 5517-5543.

Henry, C., Woodhouse, J., \& Das, S., 2002. Stability of earthquake moment tensor inversions: effect of the double-couple constraint, Tectonophysics, 356(1-3), 115-124.

Hjörleifsdóttir, V. \& Ekström, G., 2010. Effects of three-dimensional Earth structure on CMT earthquake parameters, Phys. Earth Planet. Inter., 179(3-4), 178-190.

Inoue, H., Fukao, Y., Tanabe, K., \& Ogata, Y., 1990. Whole mantle P-wave travel time tomography, Phys. Earth. Planet. Int., 59, 294-328.

Jost, M. u. \& Herrmann, R., 1989. A student's guide to and review of moment tensors, Seismological Research Letters, 60(2), 37-57.

Kanamori, H. \& Given, J. W., 1981. Use of long-period surface waves for rapid determination of earthquake-source parameters, Physics of the Earth and Planetary interiors, 27(1), 8-31.

Kennett, B. L. N., Engdahl, E. R., \& Buland, R., 1995. Constraints on seismic velocities in the Earth from traveltimes., Geophys. J. Int., 122, 108-124.

Knopoff, L. \& Randall, M. J., 1970. The compensated linear-vector dipole: A possible mechanism for deep earthquakes, Journal of Geophysical Research, 75(26), 4957-4963.

Komatitsch, D. \& Tromp, J., 1999. Introduction to the spectral element method for three-dimensional seismic wave propagation, Geophys. J. Int., 139, 806-822.

Krieger, L. \& Heimann, S., 2012. MoPaD-moment tensor plotting and decomposition: A tool for graphical and numerical analysis of seismic moment tensors, Seismological Research Letters, 83(3), 589-595.

Krischer, L., Fichtner, A., Žukauskaite, S., \& Igel, H., 2015. Large-scale seismic inversion framework, Seis. Res. Lett., 86, 1198-1207.

Krischer, L., Fichtner, A., Boehm, C., \& Igel, H., 2018. Automated large-scale full seismic waveform 
inversion for north america and the north atlantic, Journal of Geophysical Research: Solid Earth, 123(7), 5902-5928.

Lekić, V. \& Romanowicz, B., 2011. Inferring upper-mantle structure by full waveform tomography with the spectral-element method, Geophys. J. Int., 185, 799-831.

Liu, Q., Polet, J., Komatitsch, D., \& Tromp, J., 2004. Spectral-element moment tensor inversions for earthquakes in southern California, Bulletin of the Seismological Society of America, 94(5), 1748-1761.

Lu, C. \& Grand, S. P., 2016. The effect of subducting slabs in global shear wave tomography, Geophysical Journal International, 205(2), 1074-1085.

Luo, Y. \& Schuster, G. T., 1991. Wave-equation traveltime inversion., Geophysics, 56, 645-653.

Ma, Z. \& Masters, G., 2015. Effect of earthquake locations on Rayleigh wave azimuthal anisotropy models, Geophysical Journal International, 203(2), 1319-1333.

Ma, Z., Masters, G., Laske, G., \& Pasyanos, M., 2014. A comprehensive dispersion model of surface wave phase and group velocity for the globe, Geophysical Journal International, 199(1), 113-135.

Maceira, M. \& Ammon, C. J., 2009. Joint inversion of surface wave velocity and gravity observations and its application to central Asian basins shear velocity structure, J. Geophys. Res., 114, doi:1029/2007JB005157.

Masse, R. \& Needham, R., 1989. Neic-the national earthquake information center, Earthquakes \& Volcanoes (USGS), 21(1), 4-44.

Moulik, P. \& Ekström, G., 2016. The relationships between large-scale variations in shear velocity, density, and compressional velocity in the Earth's mantle, J. Geophys. Res., 121(4), 2737-2771.

Nolet, G., 2008. A breviary of seismic tomography, Cambridge University Press, Cambridge, UK.

Patton, H. J. \& Randall, G. E., 2002. On the causes of biased estimates of seismic moment for earthquakes in central Asia, Journal of Geophysical Research: Solid Earth, 107(B11), ESE-8.

Pavlis, G. L. \& Booker, J. R., 1980. The mixed discrete-continuous inverse problem: Application to the simultaneous determination of earthquake hypocenters and velocity structure, Journal of Geophysical Research: Solid Earth, 85(B9), 4801-4810.

Pilia, S., Rawlinson, N., Direen, N. G., Cummins, P. R., \& Balfour, N., 2013. Structural controls on localized intraplate deformation and seismicity in southern australia: Insights from local earthquake tomography of the flinders ranges, Journal of Geophysical Research: Solid Earth, 118(5), 2176-2190.

Seriani, G. \& Priolo, E., 1994. Spectral element method for acoustic wave simulation in heterogeneous media, Finite Elements in Analysis and Design, 16, 337-348.

Shearer, P. M., 2001. Improving global seismic event locations using source-receiver reciprocity, Bulletin of the Seismological Society of America, 91(3), 594-603.

Shearer, P. M., 2019. Introduction to Seismology, Cambridge University Press, 3rd edn.

Shuler, A., Nettles, M., \& Ekström, G., 2013. Global observation of vertical-clvd earthquakes at active 
volcanoes, Journal of Geophysical Research: Solid Earth, 118(1), 138-164.

Simmons, N. A., Forte, A. M., Boschi, L., \& Grand, S. P., 2010. GyPSuM: A joint tomography model of mantle density and seismic wave speeds, J. Geophys. Res., 115(B12), n/a-n/a.

Simons, M., Minson, S. E., Sladen, A., Ortega, F., Jiang, J., Owen, S. E., Meng, L., Ampuero, J.-P., Wei, S., Chu, R., Helmberger, D. V., Kanamori, H., Hetland, E., Moore, A. W., \& Webb, F. H., 2011. The 2011 magnitude 9.0 tohoku-oki earthquake: Mosaicking the megathrust from seconds to centuries, Science, 332(6036), 1421-1425.

Simute, S., Steptoe, H., Cobden, L., Gokhberg, A., \& Fichtner, A., 2016. Full-waveform inversion of the Japanese Islands region, J. Geophys. Res., 121(5), 3722-3741.

Sipkin, S. A., 2002. 50 - usgs earthquake moment tensor catalog, in International Handbook of Earthquake and Engineering Seismology, Part A, vol. 81 of International Geophysics, pp. 823-825, eds Lee, W. H., Kanamori, H., Jennings, P. C., \& Kisslinger, C., Academic Press.

Smith, G. P. \& Ekström, G., 1996. Improving teleseismic event locations using a three-dimensional Earth model, Bull. Seismol. Soc. Am., 86(3), 788-796.

Smith, G. P. \& Ekström, G., 1997. Interpretation of earthquake epicenter and CMT centroid locations, in terms of rupture length and direction, Phys. Earth Planet. Inter., 102(1-2), 123-132.

Somerville, P., Irikura, K., Graves, R., Sawada, S., Wald, D., Abrahamson, N., Iwasaki, Y., Kagawa, T., Smith, N., \& Kowada, A., 1999. Characterizing crustal earthquake slip models for the prediction of strong ground motion, Seismological Research Letters, 70(1), 59-80.

Spencer, C. \& Gubbins, D., 1980. Travel-time inversion for simultaneous earthquake location and velocity structure determination in laterally varying media, Geophys. J. Int., 63(1), 95-116.

Stein, S. \& Pelayo, A., 1991. Seismological constraints on stress in the oceanic lithosphere, Philosophical Transactions of the Royal Society of London. Series A: Physical and Engineering Sciences, 337(1645), $53-72$.

Storchak, D. A., Di Giacomo, D., Engdahl, E., Harris, J., Bondár, I., Lee, W. H., Bormann, P., \& Villaseñor, A., 2015. The ISC-GEM global instrumental earthquake catalogue (1900-2009): introduction, Physics of the Earth and Planetary Interiors, 239, 48-63.

Strasser, F. O., Arango, M., \& Bommer, J. J., 2010. Scaling of the source dimensions of interface and intraslab subduction-zone earthquakes with moment magnitude, Seismological Research Letters, 81(6), 941-950.

Tanioka, Y. \& Ruff, L. J., 1997. Source time functions, Seismological Research Letters, 68(3), 386-400.

Tape, C., Liu, Q., Maggi, A., \& Tromp, J., 2009. Adjoint tomography of the southern California crust., Science, 325, 988-992.

Thrastarson, S., van Herwaarden, D.-P., Krischer, L., \& Fichtner, A., 2021. Lasif: Large-scale seismic inversion framework - an updated version, Earth ArXiV preprint. 
Thurber, C. H., 1983. Earthquake locations and three-dimensional crustal structure in the Coyote Lake area, central California, Journal of Geophysical Research: Solid Earth, 88(B10), 8226-8236.

Thurber, C. H., 1992. Hypocenter-velocity structure coupling in local earthquake tomography, Physics of the Earth and Planetary Interiors, 75(1-3), 55-62.

Tondi, R., Achauer, U., Landes, M., Davi, R., \& Besutiu, L., 2009. Unveiling seismic and density structure beneath the Vrancea seismogenic zone, Romania, J. Geophys. Res., 141, doi:10.1029/2008JB005992.

Udías, A., Vallina, A. U., Madariaga, R., \& Buforn, E., 2014. Source mechanisms of earthquakes: theory and practice, Cambridge University Press.

Ulrich, T., Gabriel, A.-A., Ampuero, J.-P., \& Xu, W., 2019. Dynamic viability of the $2016 \mathrm{Mw} 7.8$ Kaikōura earthquake cascade on weak crustal faults, Nature communications, 10(1), 1-16.

Valentine, A. P. \& Trampert, J., 2012. Assessing the uncertainties on seismic source parameters: Towards realistic error estimates for centroid-moment-tensor determinations, Physics of the Earth and Planetary Interiors, 210, 36-49.

Valentine, A. P. \& Woodhouse, J. H., 2010. Reducing errors in seismic tomography: combined inversion for sources and structure, Geophysical Journal International, 180(2), 847-857.

Vallée, M., Charléty, J., Ferreira, A. M., Delouis, B., \& Vergoz, J., 2011. SCARDEC: a new technique for the rapid determination of seismic moment magnitude, focal mechanism and source time functions for large earthquakes using body-wave deconvolution, Geophysical Journal International, 184(1), 338-358. van Herwaarden, D. P., Boehm, C., Afanasiev, M., Thrastarson, S., Krischer, L., Trampert, J., \& Fichtner, A., 2020. Accelerated full-waveform inversion using dynamic mini-batches, Geophysical Journal International, 221(2), 1427-1438.

Vavryčuk, V., 2015. Moment tensor decompositions revisited, Journal of Seismology, 19(1), 231-252.

Wells, D. L. \& Coppersmith, K. J., 1994. New empirical relationships among magnitude, rupture length, rupture width, rupture area, and surface displacement, Bulletin of the seismological Society of America, 84(4), 974-1002.

Wimpenny, S. \& Watson, C. S., 2021. gWFM: A global catalog of moderate-magnitude earthquakes studied using teleseismic body waves, Seismological Society of America, 92(1), 212-226.

Wollherr, S., Gabriel, A.-A., \& Mai, P. M., 2019. Landers 1992 “reloaded”: Integrative dynamic earthquake rupture modeling, Journal of Geophysical Research: Solid Earth, 124(7), 6666-6702.

Xu, W., Dutta, R., \& Jónsson, S., 2015. Identifying active faults by improving earthquake locations with InSAR data and bayesian estimation: The 2004 tabuk (saudi arabia) earthquake sequence, Bulletin of the Seismological Society of America, 105(2A), 765-775.

Yamaya, L., Borgeaud, A. F., Kawai, K., Geller, R. J., \& Konishi, K., 2018. Effects of redetermination of source time functions on the 3-D velocity structure inferred by waveform inversion, Physics of the Earth and Planetary Interiors, 282, 117-143. 


\section{$44 \quad$ N.A.Blom, N. Rawlinson}

Yuan, Y. O., Simons, F. J., \& Bozda ğ, E., 2015. Multiscale adjoint waveform tomography for surface and body waves, Geophysics, 80(5), R281-R302.

Zenonos, A., De Siena, L., Widiyantoro, S., \& Rawlinson, N., 2019. P and S wave travel time tomography of the SE Asia-Australia collision zone, Phys. Earth Planet. Inter., 293, 106267.

Zhao, L., Chen, P., \& Jordan, T. H., 2006. Strain Green's tensors, reciprocity, and their applications to seismic source and structure studies, Bulletin of the Seismological Society of America, 96(5), 1753-1763.

Zhu, H., Bozdağ, E., Peter, D., \& Tromp, J., 2012. Structure of the European upper mantle revealed by adjoint tomography, Nat. Geosc., 5, 493-498. 
tomography 


\title{
Mitigating the effect of errors in earthquake parameters on seismic / (adjoint) waveform
}

\author{
Nienke Blom, Nicholas Rawlinson
}

September 2, 2021

This Supplementary Material contains supporting material and figures for the manuscript "Mitigating the effect of errors in earthquake parameters on seismic (waveform) tomography". We show an example of horizontal and depth discrepancies reported by different catalogues (Section S1), the window selection procedure (Section S2), some example radiation patterns (Section S3) as well as additional synthetic inversions that we have performed (Section S5)

\section{S1 An example of horizontal and depth errors}

Figure S1 gives an example of the different horizontal and depth positions reported by different catalogues. Although this is not a comparison with any sort of 'ground truth', it gives an indication of the error size that can be expected for these parameters. It furthermore demonstrates how systematic biases in event location can exist, something which is especially likely in areas displaying strong lateral heterogeneity such as subduction zones (Shearer, 2001; Hjörleifsdóttir and Ekström, 2010; Lu and Grand, 2016). Here, this is demonstrated for the North Chilean subduction zone, using a dataset collected by Craig (2019). Comparing horizontal locations from the GCMT catalogue with those from ISC-EHB, EHB and NEIC catalogues, a systematic trench-perpendicular shift is visible beween the two, typically in the order of $20-40 \mathrm{~km}$ (Figure S1b). Given the magnitudes of the events (typically between $\mathrm{M}_{W} 5$ and 6 ), this is unlikely to be a result of the rupture size, and is more likely caused by the different Earth models used.

Figure Figure S1c and d compare depths from the GCMT catalogue with those from the local catalogue derived by Craig (2019), who uses a semi-automated stacking procedure to determine accurate earthquake depths based on multiple depth phases and an accurate local velocity model. The systematic depth shift is typically 5-15 km, with GCMT generally giving deeper values. The refined catalogue of Craig (2019) shows significantly less scatter, indicating that the results are strongly self-consistent.

\section{S2 Window selection procedure}

For all measurements used in this work (both in the 'forward' perturbation analysis and in the toy inversions) a standardised window selection procedure is followed. This is done in order to be able to compare results from different tests in an unambiguous way. For each single trace, and on every component, two windows are selected:

- The body wave window is selected between the TauP predicted arrival times (Crotwell et al., 1999) of the first $\mathrm{P}$ and the first $\mathrm{S}$ wave (which are always shown in the perturbation analyses of Section 5 as a red and a green line, respectively).

- The surface wave window is selected starting at the TauP predicted $\mathrm{S}$ arrival time and is always $150 \mathrm{~s}$ in length.

This procedure has a number of consequences.

- The body wave window is in some cases very short, namely for stations that are very close to the source. If it is significantly shorter than a period, very little meaning can be attached to the measurements that are made on it.

- These windows are selected regardless of the similarity of the traces, a trait on which both the timefrequency phase difference measurement and the cross-correlation timeshift rely. Some of the measurements - in particular those at or close to nodal planes - therefore yield unrealistic values. 

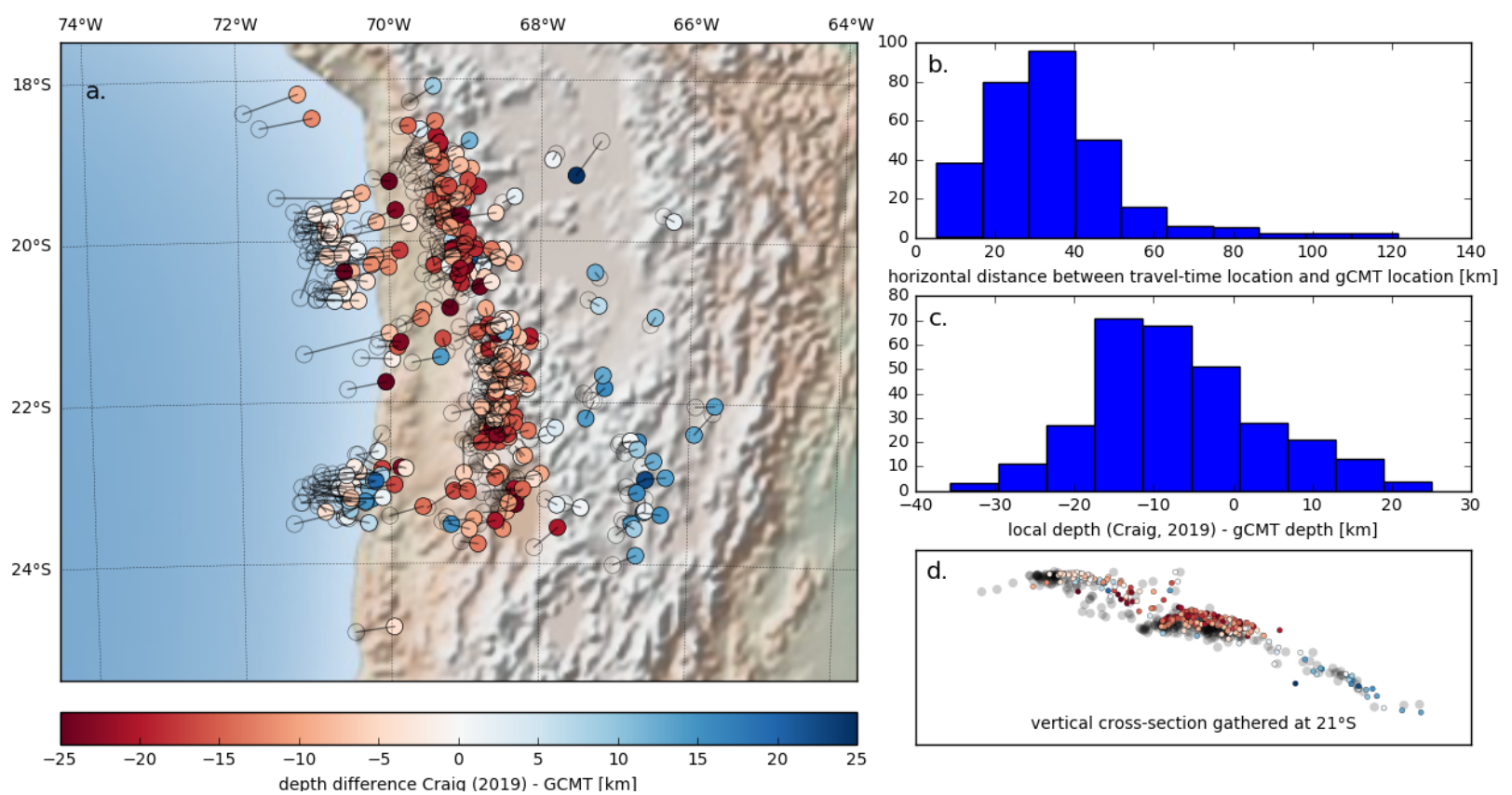

Figure S1: A comparsion between GCMT-derived and travel-time derived 3-D earthquake locations in the North Chilean subduction zone. (a) Map comparing GCMT-derived and travel-time derived earthquake locations. Open circles are GCMT locations, coloured circles represent the travel-time derived locations. Depth shifts (Craig (2019)-GCMT) are colour coded. For both, a systematic pattern is visible. (b) Histogram of horizontal distance between GCMT locations and locations reported by local travel-time studies. Most of the events are displaced by 20-40 km. (c) Histogram of depth differences between GCMT depth and depths derived using accurate depth-phase stacking (Craig, 2019). (d) Vertical cross-section of event locations. GCMT locations are plotted in black, large circles, travel-time locations are coloured by the difference between the GCMT depth and the depth derived using the depth-phase method of Craig (2019). In the latter dataset, there is much less scatter. 


\section{S3 Radiation patterns}

Figure S2 demonstrates how trace amplitude varies between components and wave types for two different events: a N-S/E-W striking strike-slip fault, and a N-S striking thrust fault. These radiation patterns give insight into where measurements made on seismograms are likely to be stable, and where they are easily affected by source errors and noise.
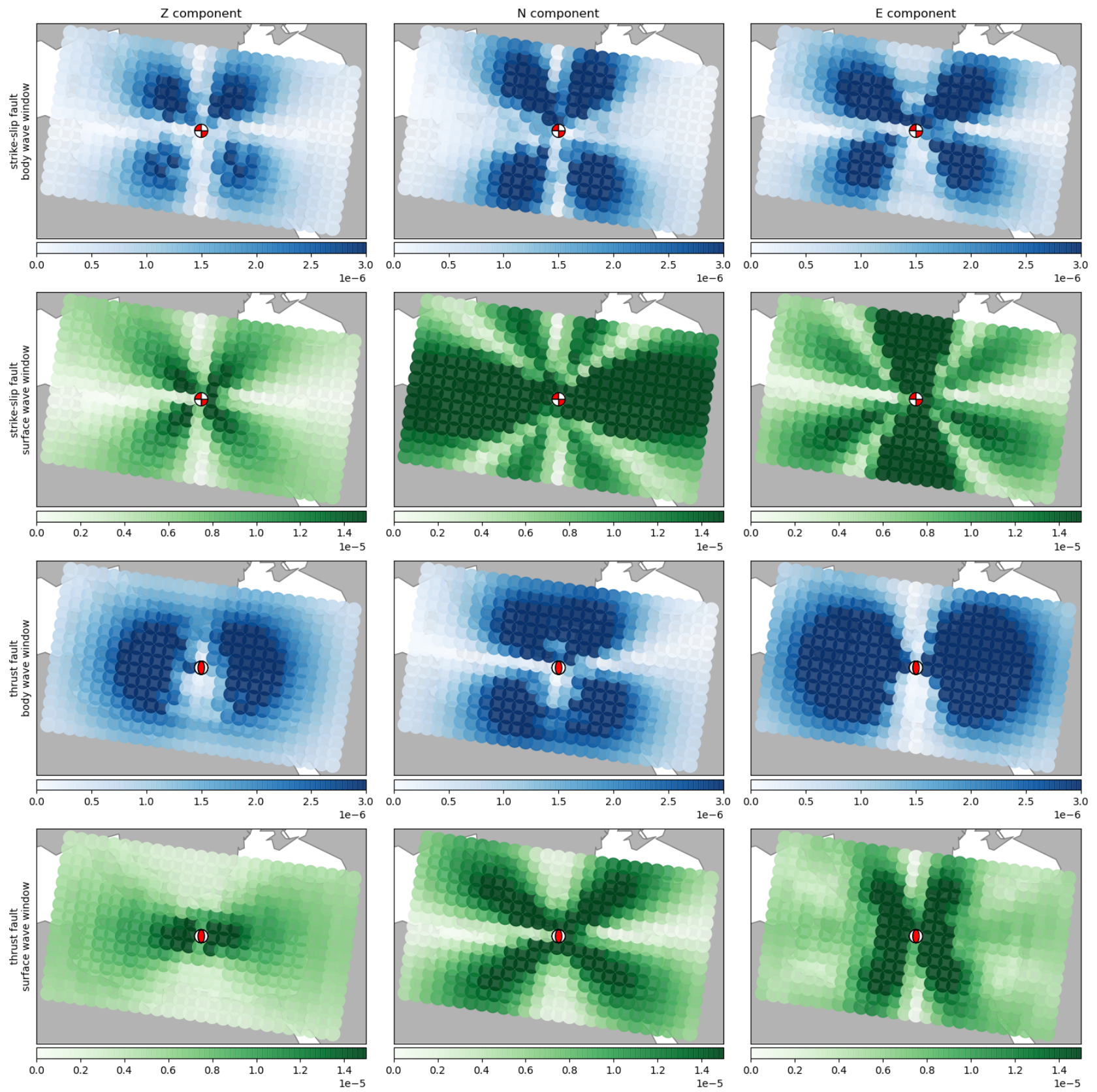

Figure S2: The spatial radiation of energy within each of the window types and for every component for a strike-slip and a thrust fault. Plotted here is the maximum amplitude recorded on the trace within the specified window. These radiation patterns were computed for the 1-D isotropic model PREM (Dziewoński and Anderson, 1981).

\section{S4 Perturbation study: additional results}

\section{S4.1 Deep events}

All results in the main manuscript are shown for a shallow event at $10 \mathrm{~km}$ depth. Here, we show the equivalent maps for an event located at $100 \mathrm{~km}$ depth (Figures S3 and S4). Results are laregely the the same (see also 
Figure 4 in the main manuscript), although the area showing near-source effects is larger for a deeper event.
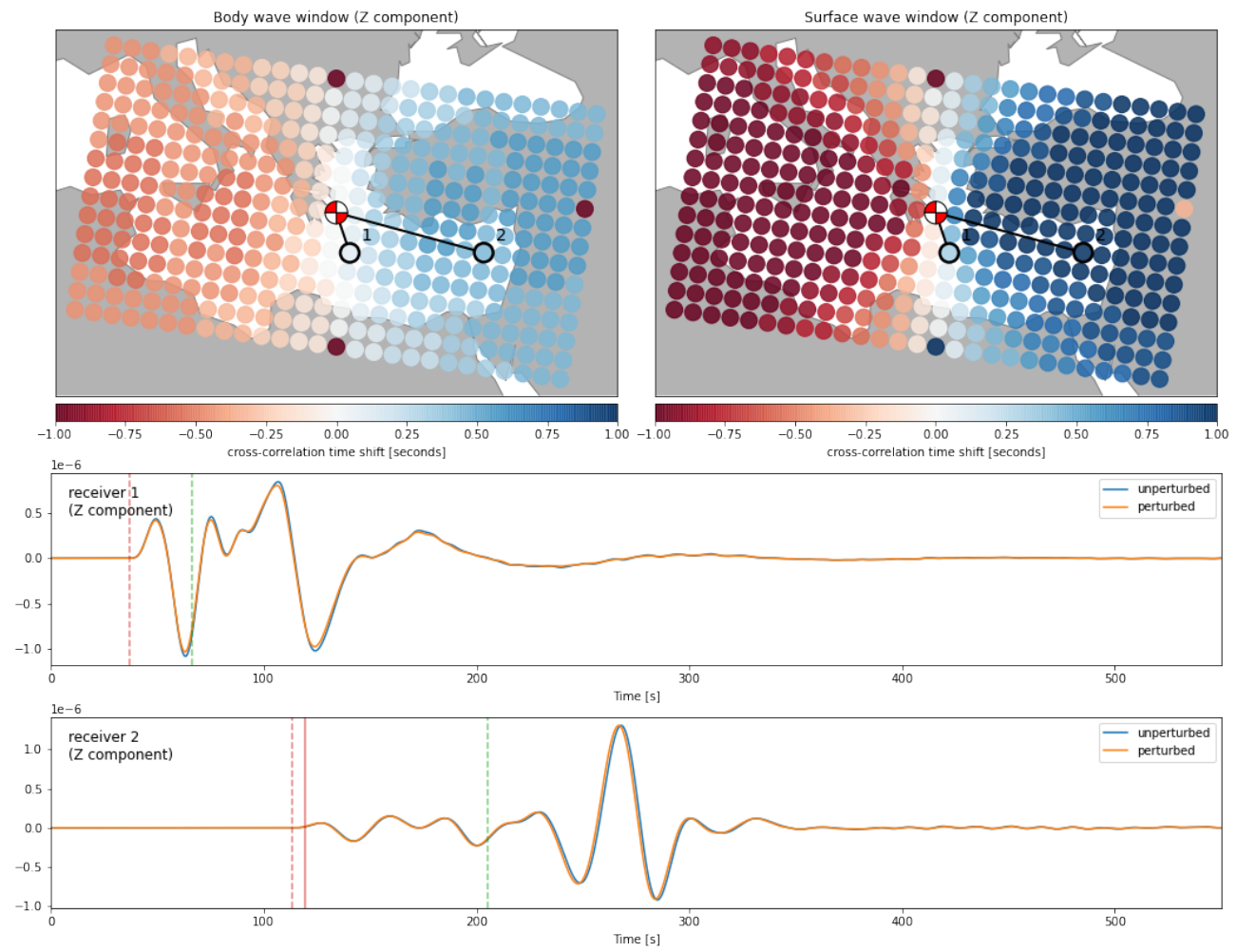

Figure S3: The effect of an eastward shift of the source by $5 \mathrm{~km}$ on seismograms in the frequency band of 28-150 s. Recorded here are cross-correlation timeshifts for an event at $100 \mathrm{~km}$ depth. For full details, see the captions to Figures 2, 3, 5 and 6 in the main manuscript.

\section{S4.2 Phase shift maps}

In the main manuscript, the effect of source perturbations is visualised using cross-correlation timeshift (main manuscript, Equation 5). We here show the same maps, but for the time-frequency phase shift (main manuscript, equation 7) (Fichtner et al., 2008). Results are shown in Figures S5-S8.

\section{S5 Additional synthetic inversions}

\section{S5.1 The effect of perturbation size on inverted artefacts}

None of the measurements we make have a linear relationship with the source perturbations we study. Nevertheless, extrapolation of our small-amplitude results can be done with some caution. We demonstrate this in Figure S9 for the case of a horizontal shift. When increasing this from a $5 \mathrm{~km}$ shift to a $20 \mathrm{~km}$ shift (a reasonable value for typical earthquakes), both the amplitude of the imaged artefact and its extent increase markedly.

\section{S5.2 The effect of event depth on inverted artefacts}

The inverted artefacts depend on the depth of the event. In Figure S10 we demonstrate the difference between the effect of a horizontal perturbation and a depth perturbation for a reference event at $10 \mathrm{~km}$ (as in the main paper) and at $100 \mathrm{~km}$ depth, respectively.

\section{S5.3 The effect of the used windows for artefacts forming as a result of a dip perturbation}

The artefacts forming as a result of a thrust fault dip perturbation do not correspond to an intuitive expectation based on the visible timeshifts. We here investigate how these artefacts change if only surface wave windows are used. As Figure S11 shows, the pattern of the formed artefacts remains largely the same, although of larger amplitude. 

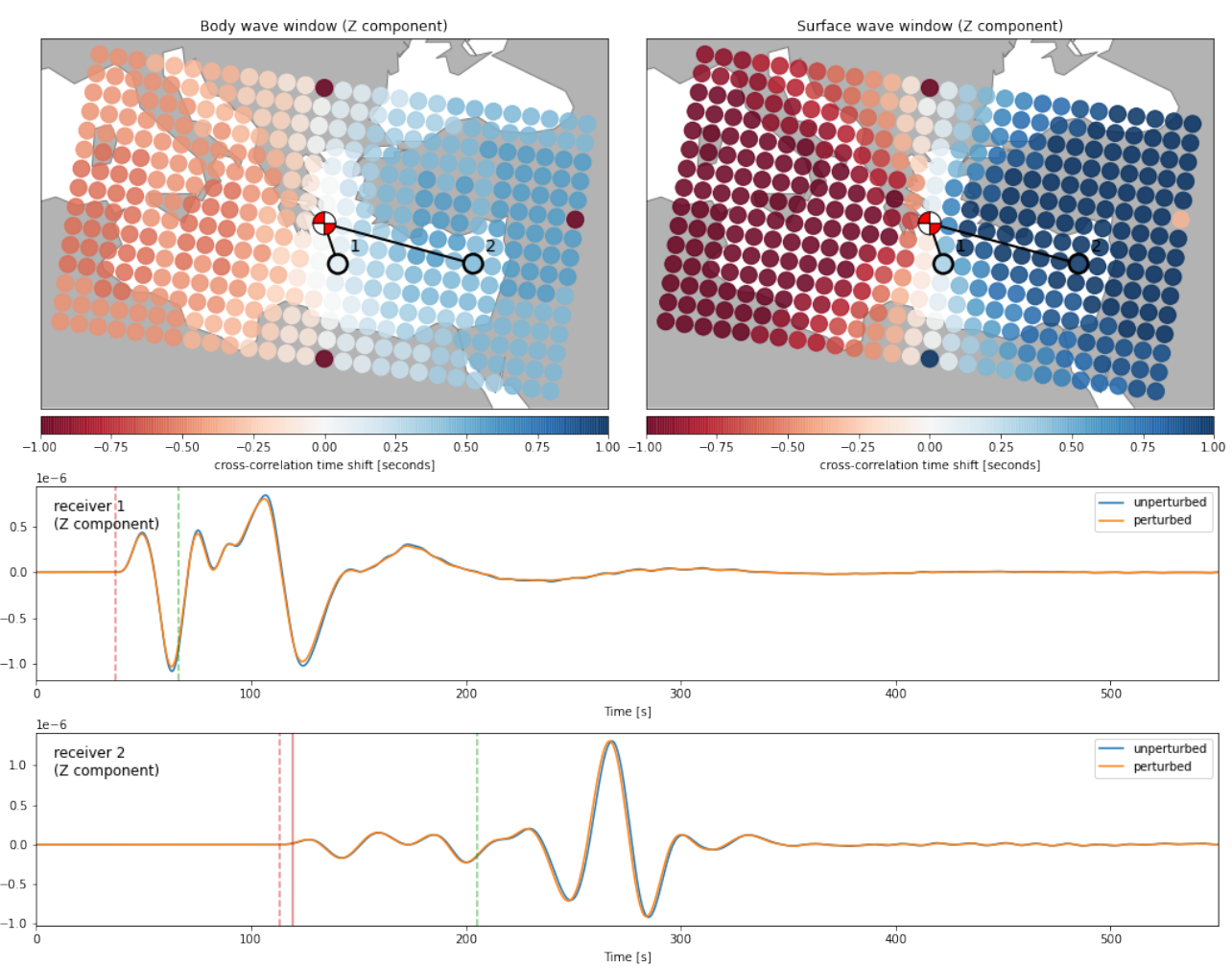

Figure S4: The effect of a $5 \mathrm{~km}$ increase in source depth on seismograms in the frequency band of $28-150 \mathrm{~s}$. Recorded here are cross-correlation timeshifts for an event at $100 \mathrm{~km}$ depth. For full details, see the captions to Figures 2, 3, 5 and 6 in the main manuscript.

\section{References}

Ahrens, J., Geveci, B., and Law, C. (2005). Paraview: An end-user tool for large data visualization. The visualization handbook.

Craig, T. (2019). Accurate depth determination for moderate-magnitude earthquakes using global teleseismic data. Journal of Geophysical Research: Solid Earth, 124(2):1759-1780.

Crotwell, H. P., Owens, T. J., and Ritsema, J. (1999). The TauP toolkit: Flexible seismic travel-time and ray-path utilities. Seismological Research Letters, 70(2):154-160.

Dziewoński, A. M. and Anderson, D. L. (1981). Preliminary reference Earth model. Phys. Earth Planet. Inter., 25:297-356.

Fichtner, A., Kennett, B. L. N., Igel, H., and Bunge, H.-P. (2008). Theoretical background for continental- and global-scale full-waveform inversion in the time-frequency domain. Geophys. J. Int., 175:665-685.

Hjörleifsdóttir, V. and Ekström, G. (2010). Effects of three-dimensional Earth structure on CMT earthquake parameters. Phys. Earth Planet. Inter., 179(3-4):178-190.

Lu, C. and Grand, S. P. (2016). The effect of subducting slabs in global shear wave tomography. Geophysical Journal International, 205(2):1074-1085.

Shearer, P. M. (2001). Improving global seismic event locations using source-receiver reciprocity. Bulletin of the Seismological Society of America, 91(3):594-603. 

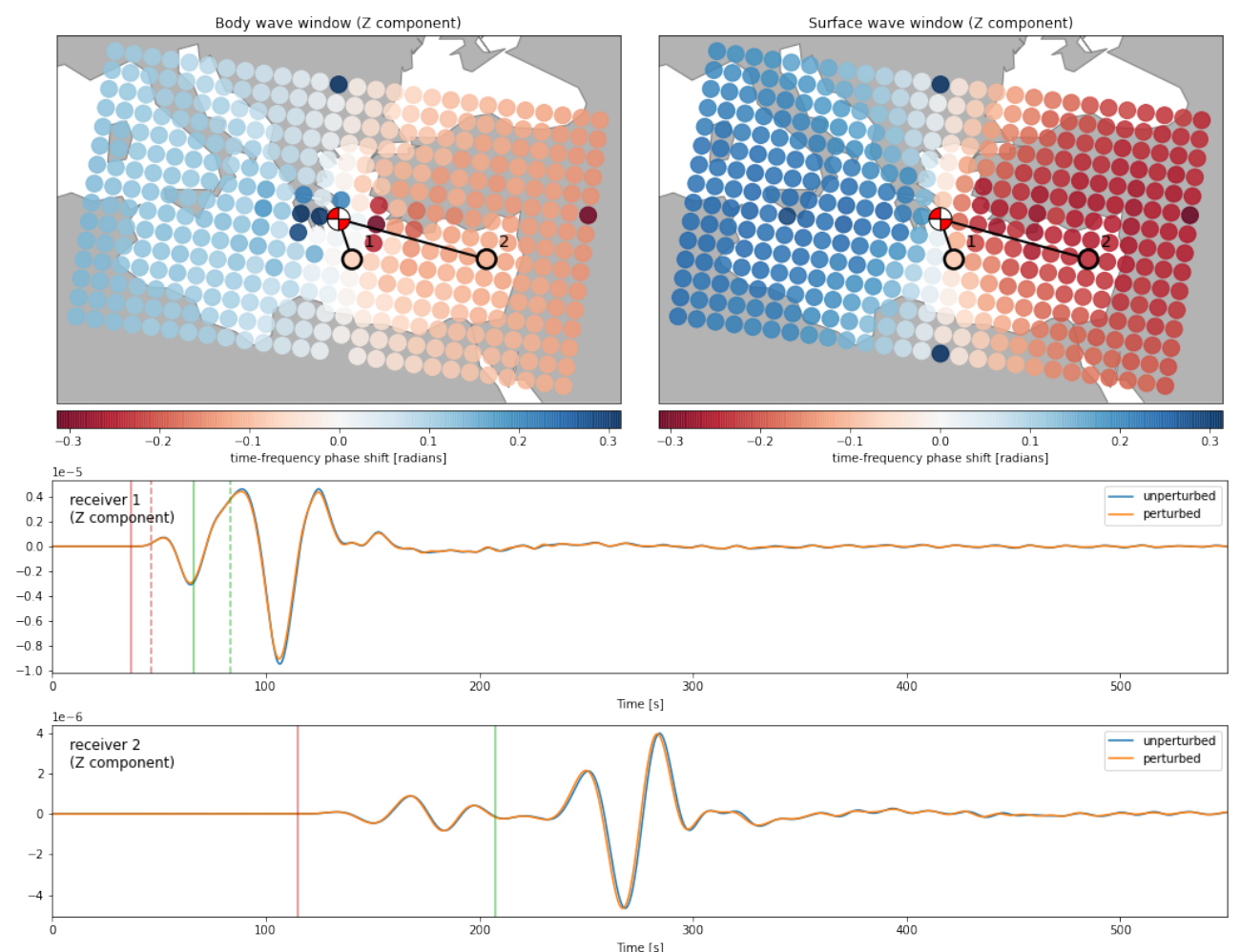

Figure S5: The effect of an eastward shift of the source by $5 \mathrm{~km}$ on seismograms in the frequency band of $28-150$ s. Recorded here are time-frequency phase shifts (Fichtner et al., 2008). For full details, see the captions to Figures 2, 3, 5 and 6 in the main manuscript.

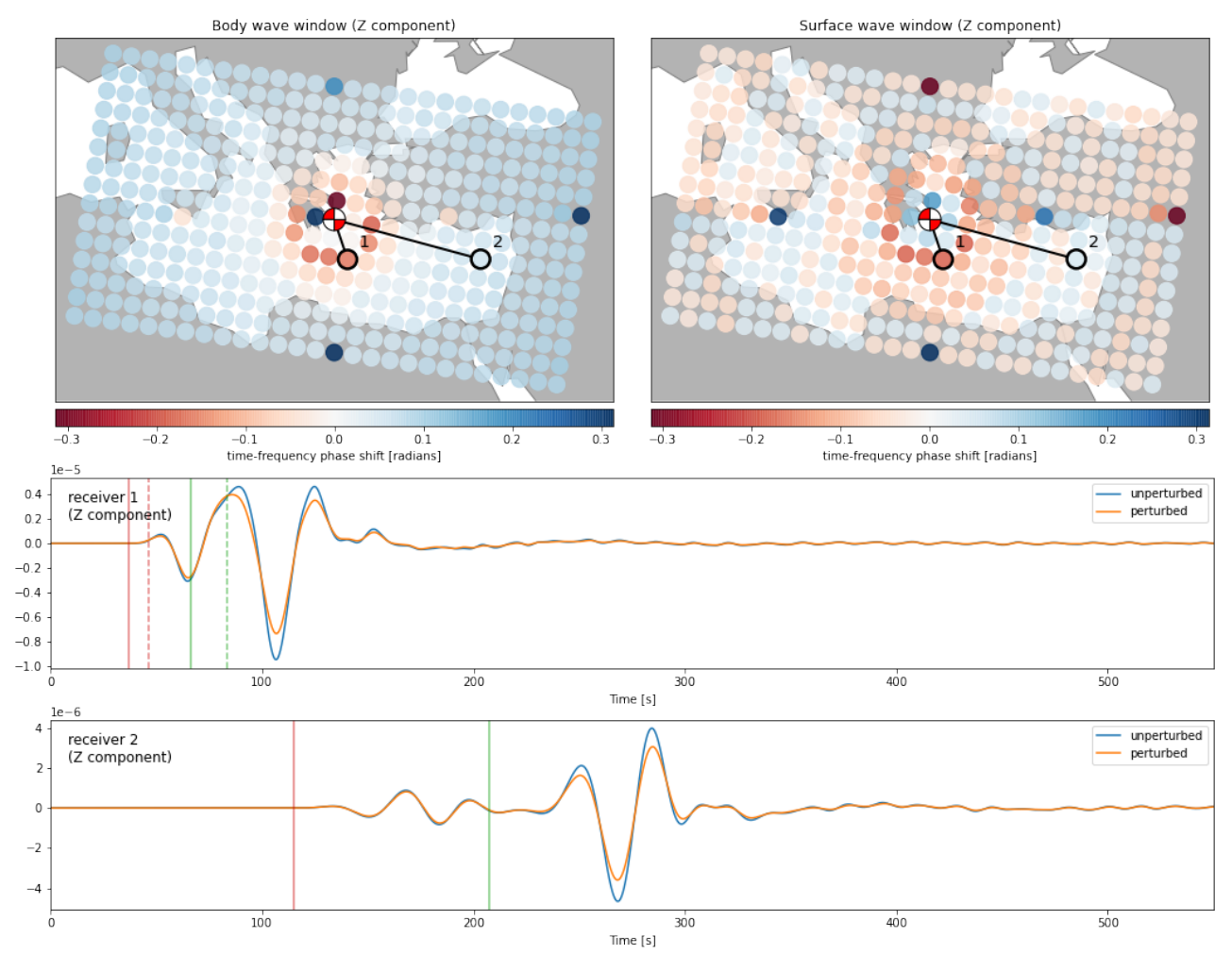

Figure S6: The effect of a $5 \mathrm{~km}$ increase in source depth on seismograms in the frequency band of $28-150 \mathrm{~s}$. Recorded here are time-frequency phase shifts (Fichtner et al., 2008). For full details, see the captions to Figures $2,3,5$ and 6 in the main manuscript. 

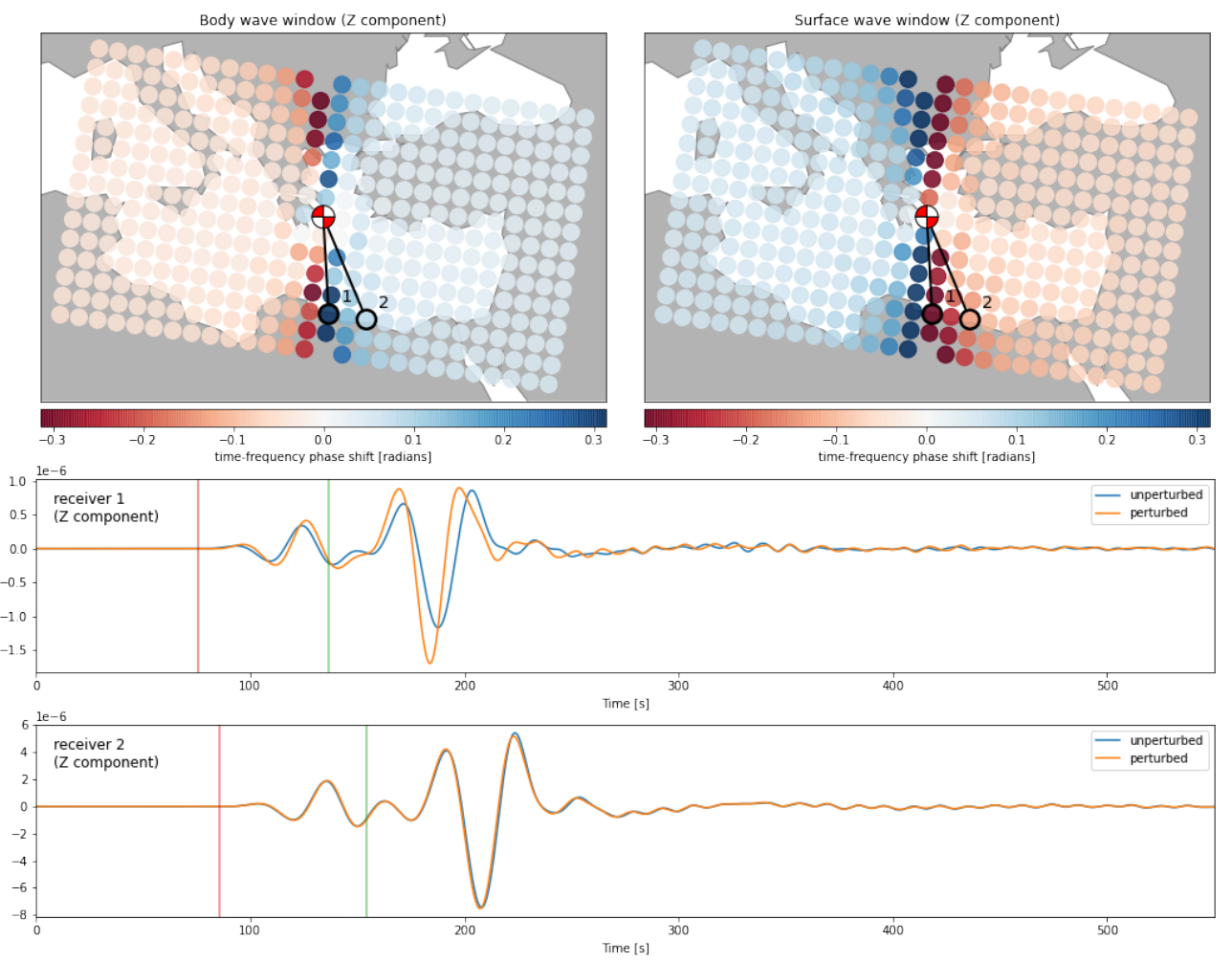

Figure S7: The effect of a $5^{\circ}$ change in the dip of the fault plane striking N-S $\left(90^{\circ}\right.$ to $85^{\circ} \mathrm{E}$ ), on seismograms in the frequency band of 28-150 s. Recorded here are time-frequency phase shifts (Fichtner et al., 2008). For full details, see the captions to Figures 2, 3, 5 and 6 in the main manuscript.
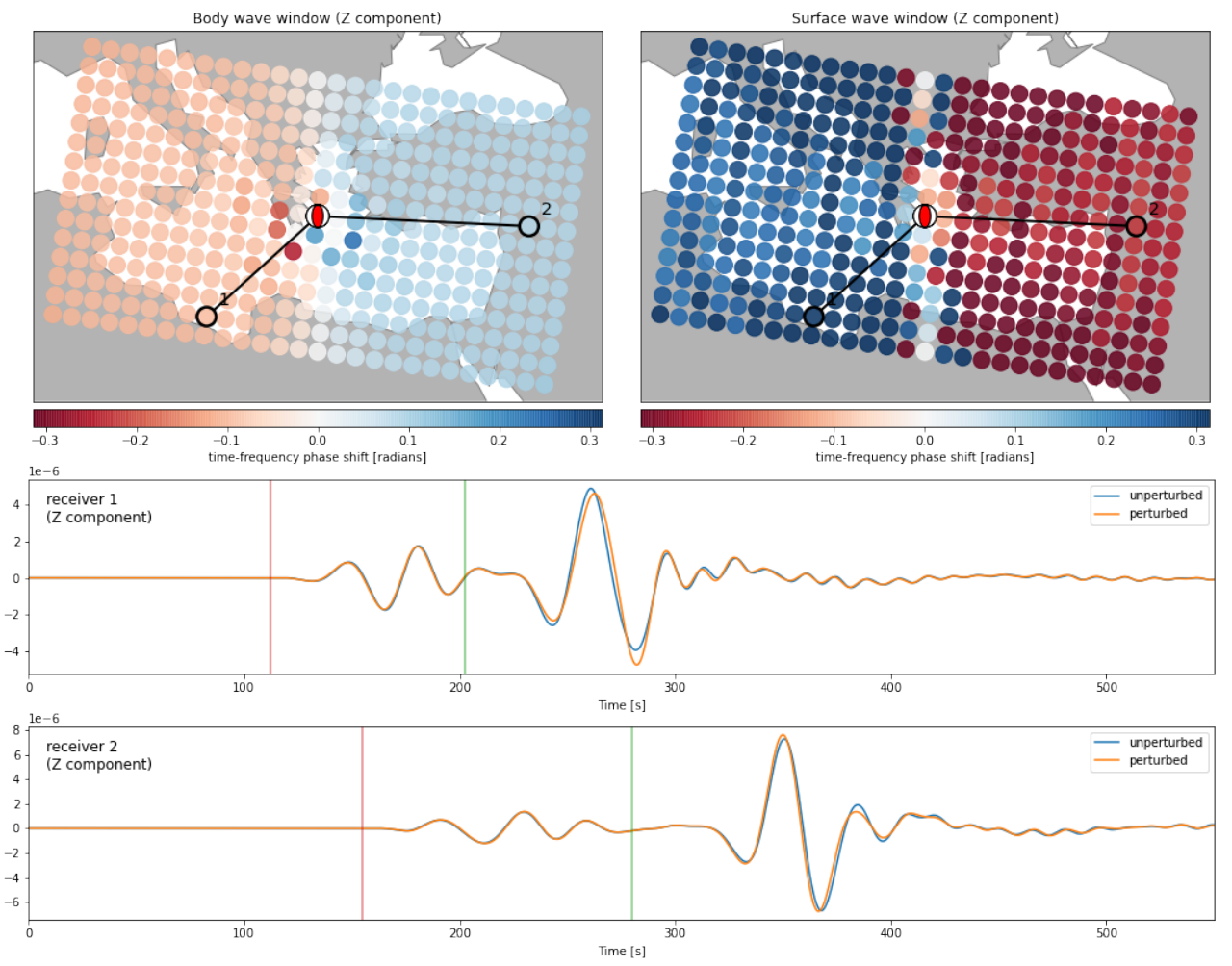

Figure S8: The effect of a $5^{\circ}$ change in dip of the fault plane of a thrust fault (from $45^{\circ} \mathrm{E}$ to $40^{\circ} \mathrm{E}$ ), for the frequency band of 28-150 s. For full details, see the captions to Figures 2, 3, 5 and 6 in the main manuscript. 

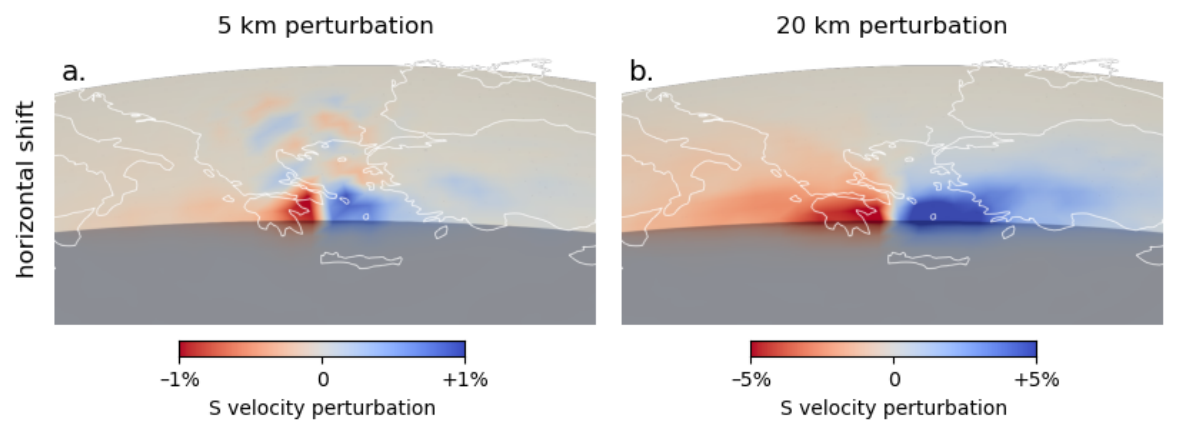

Figure S9: A demonstration of the effect of perturbation amplitude on the artefacts forming as a result of source perturbations. Results are shown for horizontal perturbations of $5 \mathrm{~km}$ (a) and $20 \mathrm{~km}$ (b). All resulting models are after 5 iterations. Note that the colour scales for both plots are different. Model sections were produced using ParaView (Ahrens et al., 2005).
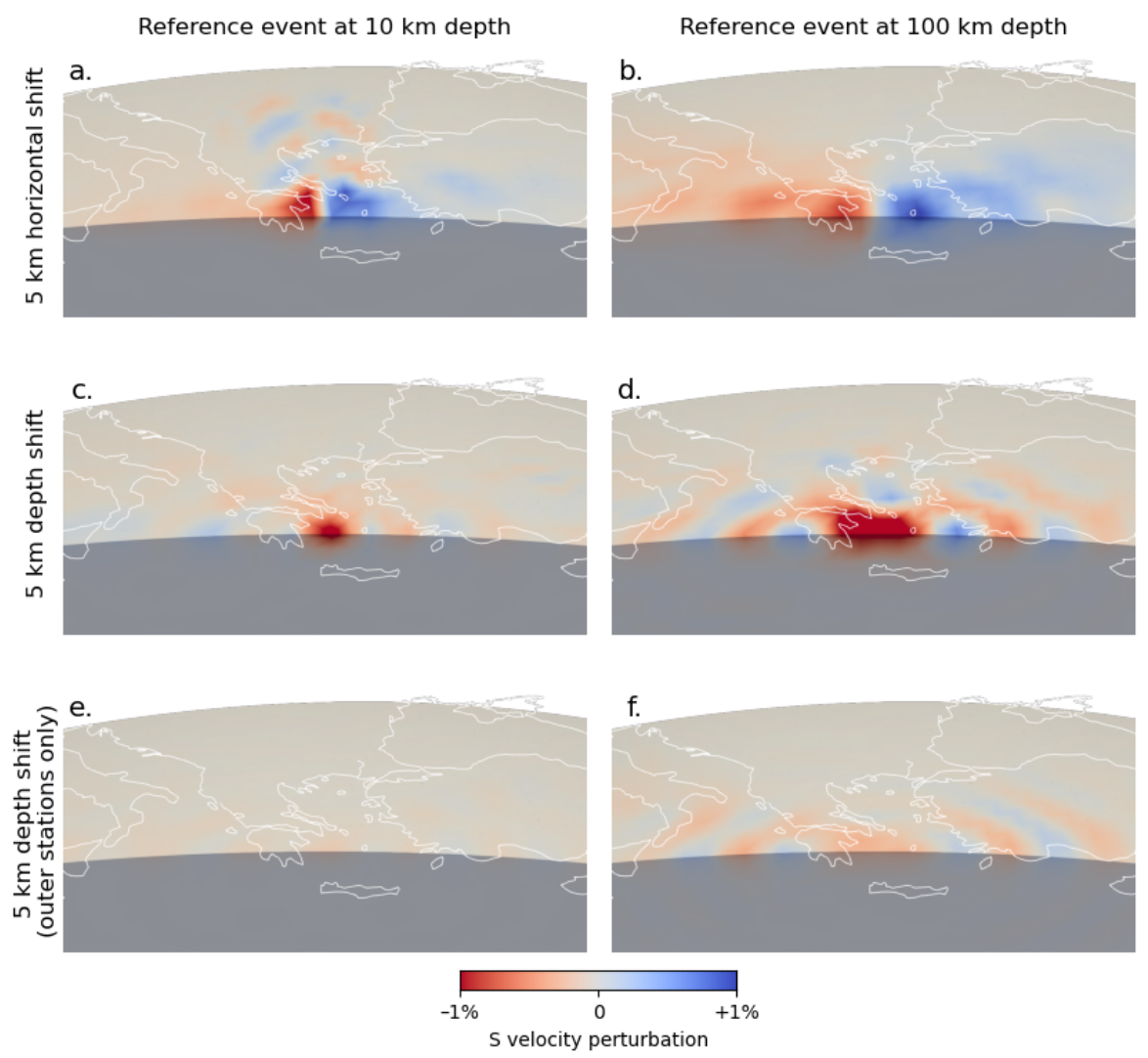

Figure S10: A demonstration of the effect of event depth on the artefacts forming as a result of source perturbations. Results are shown for a horizontal and a depth perturbation. All results are after 5 iterations. Model sections were produced using ParaView (Ahrens et al., 2005). 


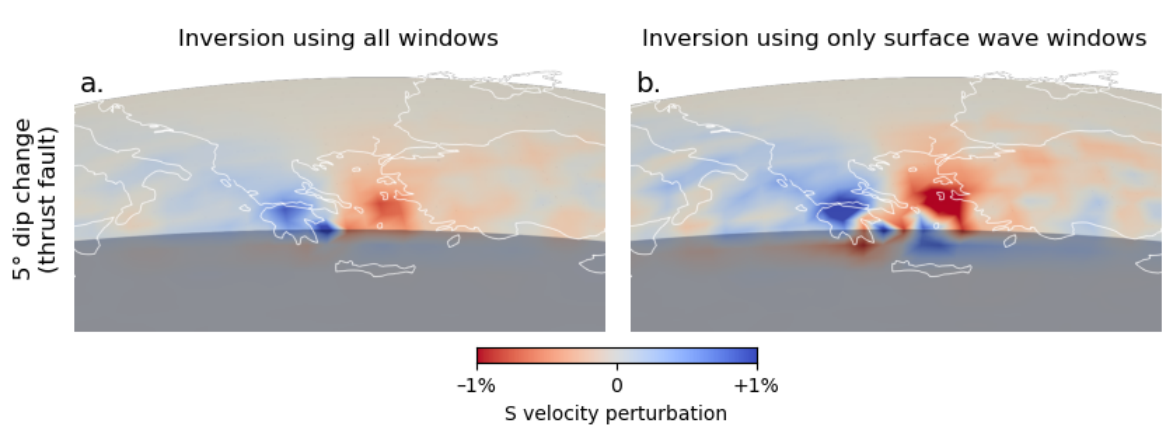

Figure S11: A demonstration of the effect of using different window types in the inversion. Results are shown for a $5^{\circ}$ perturbation in the fault plane dip on a thrust fault. Model sections were produced using ParaView (Ahrens et al., 2005). 